\title{
Upscaling of Helmholtz Equation Originating in Transmission through Metallic Gratings in Metamaterials
}

\author{
Hari Shankar Mahato \\ College of Engineering, University of Georgia, Athens, GA 30602, USA \\ Correspondence should be addressed to Hari Shankar Mahato; hsmahato@uga.edu
}

Received 28 April 2016; Accepted 26 June 2016

Academic Editor: Tongxing Li

Copyright ( 2016 Hari Shankar Mahato. This is an open access article distributed under the Creative Commons Attribution License, which permits unrestricted use, distribution, and reproduction in any medium, provided the original work is properly cited.

We investigate the transmission properties of a metallic layer with narrow slits. We consider (time-harmonic) Maxwell's equations in the $H$-parallel case with a fixed incident wavelength. We denote $\eta>0$ as the typical size of the complex structure and obtain the effective equations by letting $\eta \rightarrow 0$. For metallic permittivities with negative real part, plasmonic waves can be excited on the surfaces of the slits. For the waves to be in resonance with the height of the metallic layer, the corresponding results can be perfect transmission through the layer.

\section{Introduction}

Negative refraction of electromagnetic waves in metamaterials has become of major interest in recent years, compare $[1,2]$, especially to construct small scale optical devices for technical applications in the fields of micro- and nanooptics. Metamaterials are the materials that are not found in nature; instead they are created by the composition of several metals or plastics or both. Due to their precise shape, size, geometry, and arrangement of metals, these metamaterials are capable of influencing the electromagnetic waves by absorbing, bending, or refracting. To create the metamaterials, the composite materials are arranged in repeated (periodic) fashion with periodicity scales smaller than the wavelength of waves. Negative index metamaterial or negative index material (NIM) is a metamaterial where the refractive index (in optics theory, the refractive index of a material is a dimensionless number which describes how light propagates through that medium and is defined as the ratio $c / v$, where $c$ is the speed of light in vacuum and $v$ is the phase velocity of light in the medium) has a negative value over some frequency range when an electromagnetic wave passes through it. Negative index materials are extensively studied in the fields on optics, electromagnetics, microwave engineering, material sciences, semiconductor engineering, and several others.
In this work, we study the phenomena of light wave passing through the subwavelength metallic structure; that is, we investigate the high transmission of light wave through a metamaterial with thin holes inside it. We consider a thin metallic structure (inside a medium) with holes smaller than the wavelength of incident photon which shows the high transmission of light waves through this metallic structure. This high transmission contradicts the classical aperture theory and shows an important feature of metamaterials. To demonstrate the geometry assumed in this work, let us consider Figure 1 where the light wave emerging from a source (l.h.s of the figure) is passing through a metamaterial with negative refractive index and its image is given on the r.h.s. (cf. this figure to that of [3]).

The holes inside the metallic layer are periodically distributed with period $\eta>0$ smaller than the wavelength $\lambda$ of incident light wave. This layer can be considered as a heterogeneous or perforated media and our goal is to give a physically consistent approach to transmission properties of heterogeneous media using the techniques from homogenization theory and applied analysis. We obtain an effective (upscaled) scaterring problem where the metallic layer with holes is replaced by a homogenized structure with effective permittivity $\varepsilon_{\text {eff }}$ and permeability $\mu_{\text {eff }}$. We also obtain the tranmission coefficient $T$ in terms of incident wave number $k$ 


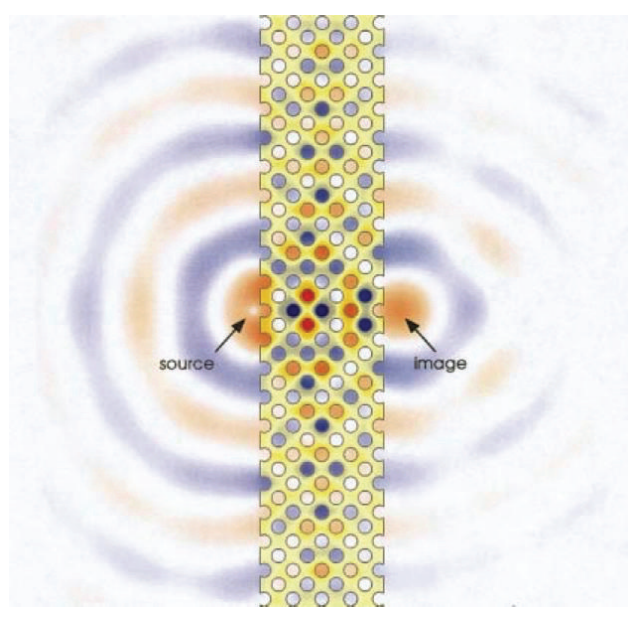

(a)

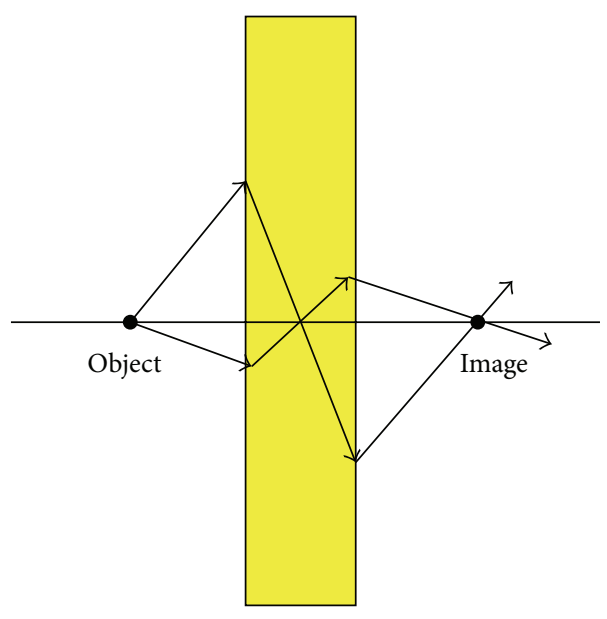

(b)

Figure 1: The light wave is originating from the left side and is getting refracted through the metallic plate and its image is on the right (this image is taken from [2]).

and incident angle $\theta$. We will see that, for lossless materials with (real) negative permittivity $\varepsilon_{\eta}$, perfect transmission $T=1$ can be obtained for every $\theta$ and suitable value for $k$. In the recent times, several significant investigations for metamaterials have been done. In [4] the connection between the high transmission and the excitation of surface plasmon polaritons has been established. The photonic band structure of the surface plasmons is evaluated numerically, In [5] the authors have calculated the transmission coefficients for the lamellar gratings, while the effect of surface plasmons on the upper and lower boundary of the layer is investigated in [6]. In [7], the effect of finite conductivity is studied. In [8], the relation between the high transmission effect and the negative index material is obtained with a fishnet like structure. A homogenization method is proposed in [9] where the author accentuates the connection between the skin depth of evanescent modes in the metallaic structure and the period of the gratings. Some results in this direction can be found in [10-13].

Two-scale convergence has proven to be a very efficient tool in homogenization theory while dealing with the problems where the underlying medium is heterogenous. The concept of two-scale convergence is first introduced by Nguetseng in [14]. This convergence criterion and the results related to it have been used extensively in the homogenization of partial differential equations; see Allaire $[15,16]$, Cioranescu and Donato [17], and Mahato and Böhm [18]. In this work too, we have used the two-scale convergence of an oscillating sequence and its gradient; see Section 1.4. At this point we would like to point out that the geometry of metallic structure in this work is generalized compared to that considered in [3]. In [3] due to the rectangular shapes of the metallic gratings, the coefficients of the effective system were determined by the help of a scalar, one-dimensional shape function $\psi: \mathbb{R} \rightarrow \mathbb{C}$ given by the hyperbolic functions; however in our work where the considered geometry is more realistic, such nice representation is not possible. To deal with this problem in this work an eigenvalue approach has been proposed and this eigenvalue problem in the unit cell $Y$ helps us to determine the effective parameters of the problem.

Although this paper can be compared with [3] in some way, the major difference in this work is that our limit function $\vec{j}_{0}$ of $\vec{j}_{\eta}=a_{\eta} \nabla u_{\eta}$ (see Section 1.5 for details) and the limit function $\vec{j}_{0}$ in [3] are totally different. In [3], the authors worked with a rectangular metallic subpart $\Sigma$ of type $(-\gamma, \gamma) \times$ $(-1 / 2,1 / 2)$ and therefore by defining a suitable test function, they have shown that the first component of $j_{0}$ vanishes and they obtain $j_{0}=\left(0, \alpha^{-1} \partial_{x_{2}} U\right)$ for $x \in R$ and $y \in Y \backslash \Sigma$. This is clearly not the case in this paper as the metallic subpart $\Sigma$ is chosen to be sinusoidal along $y_{2}$-axis due to the geometry of the metallic structure given by Figure 1 . This will lead to nonvanishing componenets of $j_{0}$ and we end up having a different $j_{0}$ compared to that in [3] and hence, we will obtain a different upscaled equation. Also no explicit representation of $\vec{j}_{0}$ can be obtained due to the geometry of $\Sigma$.

In Sections 1.1, 1.2, and 1.3, we will outline the model in detail. In Section 2, we gather some mathematical tools required to do the analysis and we state our main results. In Sections 3 and 4 we will prove the main results.

1.1. Model. We investigate the time-harmonic solutions of a Maxwell equations with a fixed wave number $k$ and the corresponding wave length $\lambda=2 \pi / k$. Let the metallic structure remain unchanged towards $x_{3}$-direction and the metallic field, denoted by $\vec{H}$, is parellel to $x_{3}$; that is, $\vec{H}=$ $(0,0, u)$, where $u: \mathbb{R}^{2} \rightarrow \mathbb{R}$.

The heterogenoeus domain $\Omega$ has a metallic structure of finite length and finite height in $\mathbb{R}^{2}$, and the slits (vacuum) are repeated periodically with a small period $\eta>0$, compare Figure 1 . The period $\eta$ is assumed to be infinitesimally small with respect to the wavelength $\lambda$. The relative permittivity of the metal is denoted by $\varepsilon$. Since the permittivity of conductors 
has large absolute values, we assume that it depends on $\eta$ and consider $\varepsilon=\varepsilon_{\eta}$. We obtain nontrivial effects due to plasmonic resonance for $\left|\varepsilon_{\eta}\right| \propto \eta^{-2}$, compare $[3,13]$. If $\Sigma_{\eta}$ denotes the matallic part in $\Omega$, we set

$$
\varepsilon_{\eta}(x):= \begin{cases}1 & \text { for } x \notin \Sigma_{\eta} \\ \frac{\varepsilon_{r}}{\eta^{2}} & \text { for } x \in \Sigma_{\eta}\end{cases}
$$

where $\varepsilon_{r} \in \mathbb{C}$. Due to ohmic losses inside the metal, $\operatorname{Im}\left(\varepsilon_{\eta}\right)$ is always assumed to be positive in a physical system which means we always take $\operatorname{Im}\left(\varepsilon_{r}\right) \geq 0$ and $\varepsilon_{r} \neq 0$. A material is called a lossless material if $\operatorname{Im}\left(\varepsilon_{r}\right)=0$. Our particular interest is to study a lossless material with negative relative permittivity; that is, $\operatorname{Im}\left(\varepsilon_{r}\right)=0$ and $\operatorname{Re}\left(\varepsilon_{r}\right)<0$. For such $\varepsilon_{r}$ transverse evanescent modes will be generated in the metal. Since $\operatorname{Re}\left(\varepsilon_{r}\right)<0$ and $\operatorname{Im}\left(\varepsilon_{r}\right)=0$, then from (14) we have $u_{\eta}\left(x_{1}, x_{2}\right)=\left(A_{1} e^{\bar{k} x_{1}}+A_{2} e^{-\bar{k} x_{1}}\right)\left(B_{1} x_{2}+B_{2}\right)$; that is, we can obtain wave like solutions and waves cannot penetrate the metallic grating. These evanescent modes can penetrate only in a region which is given by the skin depth of order $\eta$. The evanascent mode is related to a surface plasmon solution (in this case a solution which is nonvanishing in the grating but which has exponential decay in the metal). The main aspect of the current work is to generalize the geometric structure of the metallic slab inside $\Omega$ given in [3].

1.2. Geometry. Let $\eta>0$ be a small scale parameter and $\Omega$ be the domain under investigation which is bounded in $\mathbb{R}^{2}$. Let $Y:=(-1 / 2,1 / 2) \times(-1 / 2,1 / 2)$ be the representative unit cell in $\mathbb{R}^{2}$ and $\Sigma$ be an open set in $Y$ such that $\bar{\Sigma} \subset Y$ and $Y:=\Sigma \cup(Y \backslash \Sigma)$. Let us choose $\Sigma$ in such a way that it follows a sinusoidal profile along $y_{2}$-axis; that is,

$$
\begin{aligned}
\Sigma & :=\left\{\left(y_{1}, y_{2}\right):\left|y_{2}\right| \leq \frac{1}{2}, y_{1} \in S_{\gamma}, S_{\gamma}\right. \\
& :=\left(-\frac{3 \gamma}{4}+\frac{\gamma}{4} \cos \left(2 \pi y_{2}\right), \frac{3 \gamma}{4}+\frac{\gamma}{4} \cos \left(2 \pi y_{2}\right)\right), \gamma \\
& \left.\in\left(0, \frac{1}{2}\right)\right\} .
\end{aligned}
$$

Keeping physics of the problem in mind, $\Sigma$ denotes the mettalic part which lies between the two columns of holes in the metallic structure of type introduced in Figure 1.

The relative aperture volume $\alpha=1-3 \gamma / 2 \in(1 / 4,1)$ and relative metal volume is $(3 / 2) \gamma$, where $\gamma \in(0,1 / 2)$. We define $2 N_{\eta}+1:=2 l / 2 \eta \gamma$. We assume that the compact rectangle $\bar{R}$ contains $\left(2 N_{\eta}+1\right)$ number of small rectangles of type $(n \eta-\eta \gamma, n \eta+\eta \gamma) \times(-h, 0)$, that is, of width $2 \gamma \eta$ and height $h$, which include the $\eta$-scaled versions of the metallic part $\Sigma$ (cf. Figures 2 and 3), where each $\Sigma$ is of width (3/2) $\gamma \eta$ and height $h$. The collection of these small $\eta$-scaled versions of the metallic part is the metallic domain $\Sigma_{\eta}$ and assume that the two-dimensional heterogeneous metallic structure introduced in Figure 1, denoted by $\Sigma_{\eta}$ and parellel to $x_{1}$-axis, is contained in the closure of the set $R:=(-l, l) \times(-h, 0) \subset \Omega$ with $\bar{R} \subset \Omega$; that is;

$$
\Sigma_{\eta} \subset \bigcup_{n=-N_{\eta}}^{N_{\eta}}(n \eta-\eta \gamma, n \eta+\eta \gamma) \times(-h, 0) \subset \bar{R} \subset \subset \Omega,
$$

see Figure 3.

As $\eta \rightarrow 0, N_{\eta} \rightarrow \infty$. Due to nondimensionalization, we are, however, only interested in $h=1$.

1.3. Function Spaces. Let $\theta \in[0,1]$ and $1 \leq r, s \leq \infty$ be such that $1 / r+1 / s=1$. Assume that $\Xi \in\left\{\Omega, \Sigma_{\eta}\right\}$ and $l \in \mathbb{N}_{0}$; then as usual $L^{r}(\Xi)$ and $H^{l, r}(\Xi)$ denote the Lebesgue and Sobolev spaces with their usual norms and they are denoted by $\|\cdot\|_{r}$ and $\|\cdot\|_{l, r}$. For the sake of clarity if $\phi \in L^{r}(\Xi)$, then

$$
\|\phi\|_{L^{r}(\Xi)}= \begin{cases}{\left[\int_{\Xi}|\phi(x)|^{r} d x\right]^{1 / p}} & \text { for } 1 \leq r<\infty, \\ \underset{x \in \Xi}{\operatorname{ess} \sup _{x}|\phi(x)|} & \text { for } r=\infty,\end{cases}
$$

and if $\phi \in H^{l, r}(\Xi)$, then

$$
\begin{aligned}
& \|\phi\|_{l, r}:=\|\phi\|_{H^{l, r}(\Xi)} \\
& := \begin{cases}{\left[\sum_{|\alpha| \leq l} \int_{\Xi}\left|D^{\alpha} \phi\right|^{r} d x\right]^{1 / r}} & \text { for } 1 \leq r<\infty, \\
\sum_{|\alpha| \leq l} \operatorname{ess} \sup _{x \in \Xi}\left|D^{\alpha} \phi(x)\right| & \text { for } r=\infty,\end{cases}
\end{aligned}
$$

where $\alpha=\left(\alpha_{1}, \alpha_{2}, \ldots, \alpha_{n}\right) \in \mathbb{N}^{n}$ is a multi-index, $|\alpha|=\alpha_{1}+$ $\alpha_{2}+\cdots+\alpha_{n}$, and $D^{\alpha}=\partial^{|\alpha|} / \partial_{x_{1}}^{\alpha_{1}} \partial_{x_{2}}^{\alpha_{2}} \cdots \partial_{x_{n}}^{\alpha_{n}}$. Similarly, $C^{\theta}(\bar{\Xi})$, $(\cdot, \cdot)_{\theta, r}$, and $[\cdot, \cdot]_{\theta}$ are the Hölder, real, and complex interpolation spaces, respectively, endowed with their standard norms; for definition confer $[19,20] . C_{\#}^{\alpha}(Y)$ denotes the set of all $Y$ periodic $\alpha$-times continuously differentiable functions in $y$ for $\alpha \in \mathbb{N}$. In particular, $C_{\#}(Y)$ is the space of all the $Y$ periodic continuous function in $y$. The $C^{\infty}$-spaces are as usual equipped with their maximum norm whereas the space of all continuous functions $C(\Xi)$ is furnished with supremum norm, compare in [19].

\subsection{Two-Scale Convergence}

Definition 1. A sequence of functions $\left(u^{\eta}\right)_{\eta>0}$ in $L^{r}((0, T) \times \Omega)$ is said to be two-scale convergent to a limit $u \in L^{r}((0, T) \times \Omega \times$ $Y)$ if

$$
\begin{aligned}
\lim _{\eta \rightarrow 0} & \int_{0}^{T} \int_{\Omega} u^{\eta}(t, x) \phi\left(t, x, \frac{x}{\eta}\right) d x d t \\
& =\int_{0}^{T} \int_{\Omega} \int_{Y} u(t, x, y) \phi(t, x, y) d x d t d y
\end{aligned}
$$

for all $\phi \in L^{s}\left((0, T) \times \Omega ; C_{\#}(Y)\right)$.

By $\stackrel{2}{\rightarrow}, \stackrel{w}{\rightarrow}$, and $\rightarrow$ we denote the two-scale, weak, and strong convergence of a sequence, respectively. Finally, $S=$ $(0, T)$ denotes the time interval. 


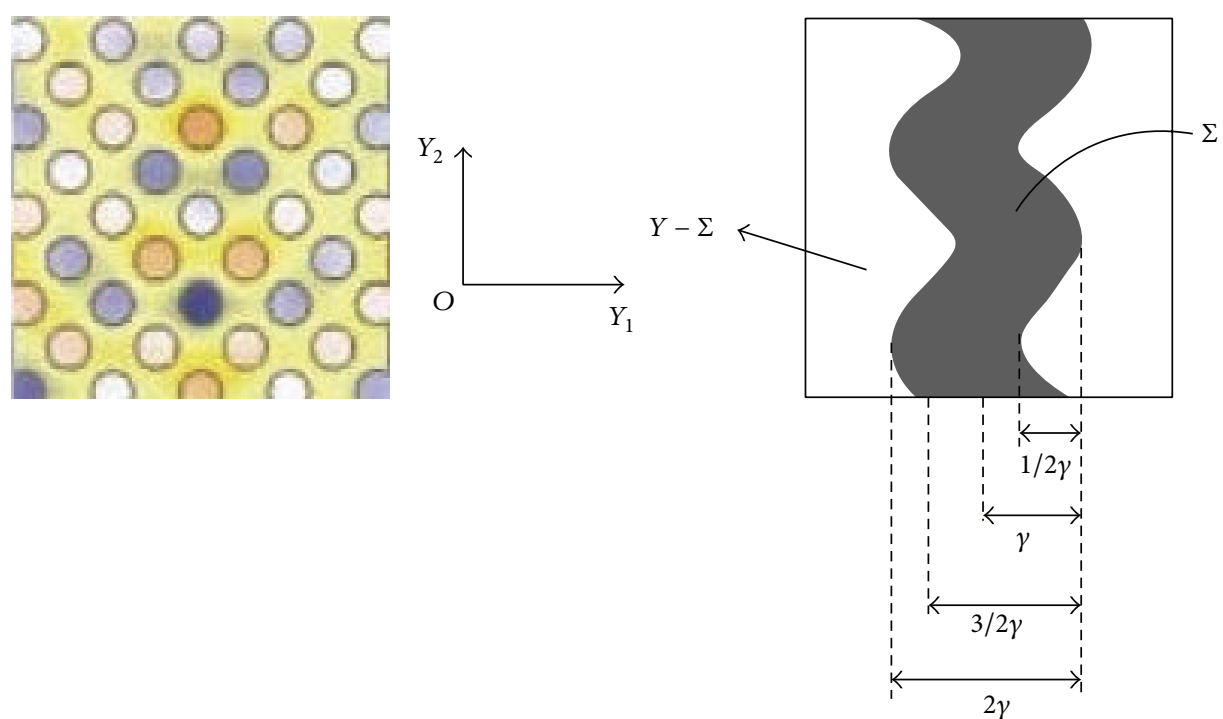

FIgURE 2: The representative cell $Y \subset \mathbb{R}^{2}$ (cf. this figure with figure 1 in [3]).

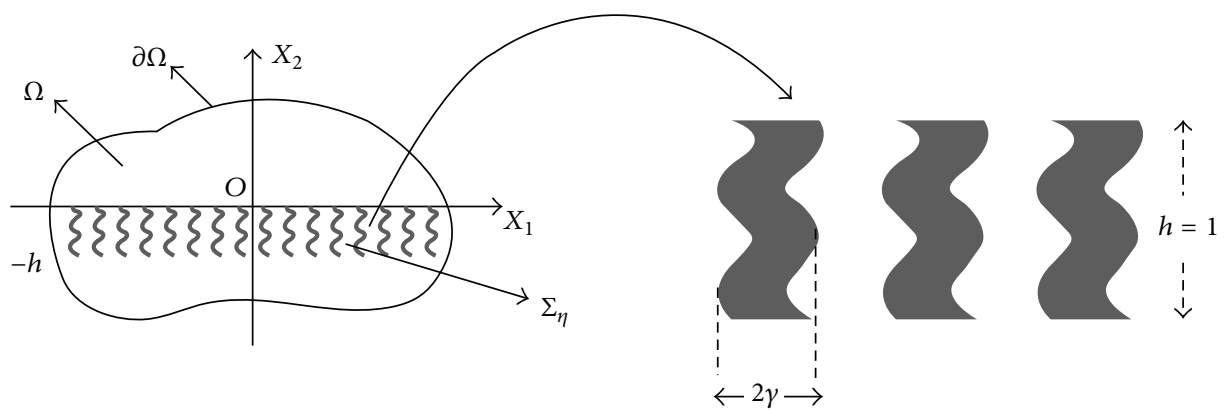

FIgURE 3: The metallic structure $\Sigma_{\eta}$ inside the domain $\Omega$.

Lemma 2 (cf. [21]). For every bounded sequence $\left(u^{\eta}\right)_{\eta>0}$ in $L^{r}(S \times \Omega)$ there exists a subsequence $\left(u^{\eta}\right)_{\eta>0}$ (still denoted by same symbol) and $u \in L^{r}((0, T) \times \Omega \times Y)$ such that $u^{\eta} \stackrel{2}{\longrightarrow} u$.

Lemma 3 (cf. [21]). Let $\left(u^{\eta}\right)_{\eta>0}$ be strongly convergent to $u \in$ $L^{r}((0, T) \times \Omega)$, and then $u^{\eta} \stackrel{2}{\rightarrow} u_{1}$, where $u_{1}(t, x, y)=u(t, x)$.

Lemma 4 (cf. [21]). Let $\left(u^{\eta}\right)_{\eta>0}$ be a sequence in $L^{r}((0, T)$; $\left.H^{1, r}(\Omega)\right)$ such that $u^{\eta} \stackrel{w}{\rightarrow} u$ in $L^{r}\left((0, T) ; H^{1, r}(\Omega)\right)$. Then $u^{\eta} \stackrel{2}{\rightarrow}$ $u$ and there exists a subsequence $\left(u^{\eta}\right)_{\eta>0}$, still denoted by same symbol, and $u_{1} \in L^{r}\left((0, T) \times \Omega ; H_{\#}^{1, r}(Y)\right)$ such that $\nabla_{x} u^{\eta} \stackrel{2}{\rightarrow}$ $\nabla_{x} u+\nabla_{y} u_{1}$.

Lemma 5. Let $\left(u^{\eta}\right)_{\eta>0}$ be a bounded sequence of functions in $L^{r}(S \times \Omega)$ such that $\eta \nabla u^{\eta}$ and $\eta^{1 / 2} \nabla u^{\eta}$ are bounded in $L^{r}(S \times$ $\Omega)^{n}$. Then there exists some functions $u, u_{1} \in L^{r}\left(S \times \Omega ; H_{\#}^{1, r}(Y)\right)$ such that $u^{\eta} \stackrel{2}{\rightarrow} u, \eta \nabla u^{\eta} \stackrel{2}{\rightarrow} \nabla_{y} u$, and $\eta^{1 / 2} \nabla u^{\eta} \stackrel{2}{\rightarrow} \nabla_{y} u_{1}$.

Proof. (i) Since $u^{\eta}$ and $\eta \nabla u^{\eta}$ are bounded sequence of functions in $L^{r}(S \times \Omega)$ and $L^{r}\left(S ; L^{r}(\Omega)\right)^{n}$, respectively, then there exists $u \in L^{r}(S \times \Omega \times Y)$ and $U \in L^{r}(S \times \Omega \times Y)^{n}$ such that $u^{\eta} \stackrel{2}{\rightarrow} u$ and $\eta \nabla u^{\eta} \stackrel{2}{\rightarrow} U$ as $\eta \rightarrow 0$. This means that, for the sequence $\eta \nabla u^{\eta}$, we have

$$
\begin{gathered}
\eta \int_{S \times \Omega} \nabla u^{\eta} \cdot \vec{\phi}\left(x, \frac{x}{\eta}\right) d x d t \stackrel{\eta \rightarrow 0}{\longrightarrow} \\
\int_{S \times \Omega \times Y} U(x, y) \cdot \vec{\phi}(x, y) d x d y d t
\end{gathered}
$$

for all $\vec{\phi} \in C_{0}^{\infty}\left(S \times \Omega ; C_{\#}^{\infty}(Y)\right)^{n}$. We integrate by parts the l.h.s, and which gives

$$
\begin{aligned}
& \lim _{\eta \rightarrow 0} \eta \int_{S \times \Omega} \nabla u^{\eta}(x) \cdot \vec{\phi}\left(x, \frac{x}{\eta}\right) d x d t \\
& =-\lim _{\eta \rightarrow 0} \int_{S \times \Omega} u^{\eta}(x) \\
& \quad \cdot\left(\eta \nabla_{x} \cdot \phi\left(x, \frac{x}{\eta}\right)+\nabla_{y} \cdot \phi\left(x, \frac{x}{\eta}\right)\right) d x d t \\
& =-\int_{S \times \Omega \times Y} u(x, y) \nabla_{y} \phi(x, y) d x d y d t \\
& =\int_{S \times \Omega \times Y} \nabla_{y} u(x, y) \phi(x, y) d x d y d t .
\end{aligned}
$$

And it follows from (7) and (8) that $U(t, x, y)=\nabla_{y} u(t, x, y)$. 
(ii) To prove the second part of the lemma, let us choose $\phi(t, x, x / \eta)=\phi_{1}(t, x)+\sqrt{\eta} \phi_{2}(t, x, x / \eta)$, where $\overrightarrow{\phi_{1}} \in C_{0}^{\infty}(S \times$ $\Omega)^{n}$ and $\overrightarrow{\phi_{1}} \in C_{0}^{\infty}\left(S \times \Omega ; C_{\#}^{\infty}(Y)\right)^{n}$. Note that the boundedness of $\eta^{1 / 2} \nabla u^{\eta}$ implies the boundedness of $\eta \nabla u^{\eta}$ in $L^{r}(S \times \Omega)$ and hence, by part (i) there exists $u \in L^{r}\left(S \times \Omega ; H_{\#}^{1, r}(Y)\right)$ such that $u^{\eta} \stackrel{2}{\rightarrow} u$ and $\eta \nabla u^{\eta} \stackrel{2}{\rightarrow} \nabla_{y} u$. Now let us assume that $\eta^{1 / 2} \nabla u^{\eta} \stackrel{2}{\rightarrow}$ $U$, and then by definition

$$
\begin{aligned}
& \lim _{\eta \rightarrow 0} \eta^{1 / 2} \int_{S \times \Omega} \nabla u^{\eta}\left(\phi_{1}(t, x)+\sqrt{\eta} \phi_{2}\left(t, x, \frac{x}{\eta}\right)\right) d x d t \\
& \quad=\lim _{\eta \rightarrow 0} \int_{S \times \Omega}\left[\eta^{1 / 2} \nabla u^{\eta} \phi_{1}(t, x)\right. \\
& \left.+\eta \nabla u^{\eta} \phi_{2}\left(t, x, \frac{x}{\eta}\right)\right] d x d t \\
& =\int_{S \times \Omega \times Y}\left[U(t, x, y) \phi_{1}(t, x)+\nabla_{y} u(t, x, y)\right. \\
& \left.\cdot \phi_{2}(t, x, y)\right] d x d y d t .
\end{aligned}
$$

We integrate by parts the 1.h.s.; then

$$
\begin{aligned}
& -\lim _{\eta \rightarrow 0} \int_{S \times \Omega}[\eta^{1 / 2} \underbrace{u^{\eta}(x) \nabla_{x} \phi_{1}(t, x)}_{\text {bounded }} \\
& +\underbrace{\eta u^{\eta}(x) \nabla_{x} \phi_{2}\left(t, x, \frac{x}{\eta}\right)}_{\text {bounded }} \\
& \left.+u^{\eta}(x) \nabla_{y} \phi_{2}\left(t, x, \frac{x}{\eta}\right)\right] d x d t \\
& =-\int_{S \times \Omega \times Y} u(t, x, y) \nabla_{y} \phi_{2}(t, x, \\
& y) d x d y d t .
\end{aligned}
$$

We compare (9) and (10) which leads us to

$$
\int_{S \times \Omega \times Y} U(t, x, y) \phi_{1}(t, x) d x d y d t=0 .
$$

Since $\phi_{1}$ is independent of $y$ and $\nabla_{y} \cdot \phi_{1}(t, x)=0$, from (11) it follows that $U$ must be the gradient of some function $u_{1} \in$ $L^{r}\left(S \times \Omega ; H_{\#}^{1, r}(Y)\right)$ such that $U(t, x, y)=\nabla_{y} u_{1}(t, x, y)$; that is, $\eta^{1 / 2} \nabla u^{\eta} \stackrel{2}{\rightarrow} \nabla_{y} u_{1}(t, x, y)$. This completes the proof.

1.5. Mathematical Formulation and Statement of the Main Results. We study the Maxwell equations in a complex geometry with highly oscillating permittivities. By $\eta$ we denote (i) the dimensionless positive scale parameter which represents the small length scale in the geometry $\Sigma_{\eta} \subset \mathbb{R}^{2}$ and (ii) the oscillations of large absolute values of the permittivity. We follow the standard nondimenionsalization techniques; for instance, see $[3,18,22]$, and so forth and from here on all the quantities considered in this work are dimensionless unless stated otherwise. For the electric field $\vec{E}_{\eta}$ and magnetic field $\vec{H}_{\eta}$, the time-harmonic Maxwell equations are

$$
\begin{aligned}
& \nabla \times \vec{E}_{\eta}=i \omega \mu_{0} \vec{H}_{\eta}, \\
& \nabla \times \vec{H}_{\eta}=-i \omega \varepsilon_{\eta} \varepsilon_{0} \vec{E}_{\eta},
\end{aligned}
$$

with fixed positive real constants $\omega, \mu_{0}$, and $\varepsilon_{0}$ denoting the frequency of the incident waves and the permeability and the permittivity of vacuum, respectively. We postulate that all the quantities are $x_{3}$-independent and the polarized magnetic field is given by $\vec{H}_{\eta}=\left(0,0, u_{\eta}\right)$, where $u_{\eta}: \Omega \subset \mathbb{R}^{2} \rightarrow \mathbb{R}$. By orthogonal property of $\vec{E}_{\eta}$ and $\vec{H}_{\eta}$, we have $\vec{E}_{\eta}=\left(E_{x, \eta}\right.$, $\left.E_{y, \eta}, 0\right)$. Then (12) reduce to

$$
\begin{aligned}
-\frac{\partial E_{x, \eta}}{\partial y}+\frac{\partial E_{y, \eta}}{\partial x} & =i \omega \mu_{0} u_{\eta}, \\
\left(\frac{\partial u}{\partial y},-\frac{\partial u}{\partial x}\right) & =-i \omega \varepsilon_{\eta} \varepsilon_{0}\left(E_{x, \eta}, E_{y, \eta}\right) .
\end{aligned}
$$

By (13), a straightforward calculation yields

$$
\nabla \cdot\left(\varepsilon_{\eta}^{-1} \nabla u_{\eta}\right)=-k^{2} u_{\eta}
$$

where we have set $k^{2}=\omega^{2} \varepsilon_{0} \mu_{0}$. We define the coefficient $a_{\eta}(x):=\varepsilon_{\eta}^{-1}(x)$ which can have a negative real part and that it vanishes in the metal as $\eta \rightarrow 0$. Thus we have the desired Helmholtz equation which we will study in this paper and is given below. We study solutions $u_{\eta} \in H_{\text {loc }}^{1}(\Omega)$ of

$$
\nabla \cdot\left(a_{\eta} \nabla u_{\eta}\right)=-k^{2} u_{\eta} \quad \text { in } \Omega,
$$

where the coefficient $a_{\eta}$ is given by

$$
a_{\eta}:=\left(\varepsilon_{\eta}\right)^{-1}= \begin{cases}1 & \text { in } \Omega \backslash \Sigma_{\eta}, \\ \eta^{2} \varepsilon_{r}^{-1} & \text { in } \Sigma_{\eta} .\end{cases}
$$

The set $\Sigma_{\eta} \subset \subset R \subset \Omega$ describes the complex geometry of the metallic inclusion in $\Omega$; see Figure 3 .

Remark 6 (scattering problem). We will investigate the effective behavior of solutions of (15) in two different cases. In the first case we will study an arbitrary bounded sequence of solutions on a bounded domain $\Omega$ while the second one concerns the scattering problem. In other words we consider (15) in whole of $\mathbb{R}^{2}$. For a given incident wave $u^{i}$, which solves $\nabla^{2} u^{i}=-k^{2} u^{i}$ in $\mathbb{R}^{2}$, we take the Sommerfeld condition as the boundary condition which says that the scattered field $u_{\eta}^{s}=u_{\eta}-u^{i}$ satisfies

$$
\partial_{r} u_{\eta}^{s}-i k u_{\eta}^{s}=o\left(r^{-1 / 2}\right)
$$

for $r=|x| \rightarrow \infty$, uniformly in the angle variable. 
Remark 7. Note that for (15) we have not given any boundary conditions; instead we have considered an arbitrary sequence of solutions; however, the uniqueness of solution of the scattering problem will be proven for every $\eta$. To state the main results, we rewrite (15) as a system:

$$
\begin{array}{r}
\nabla \cdot j_{\eta}=-k^{2} u_{\eta}, \\
j_{\eta}=a_{\eta} \nabla u_{\eta} .
\end{array}
$$

Comparing with (12), we see that $j_{\eta}$ represents (up to a factor and perhaps a rotation) the horizontal electric field $\vec{E}_{\eta}$ and since the magnetic field $\vec{H}_{\eta}(x)=\left(0,0, u_{\eta}(x)\right)$, system (18) is nothing but (12) itself.

Theorem 8 (upscaled equations). Let the matallic geometry be given by $\Sigma_{\eta}$ (Figure 3) on a domain $\Omega \subset \mathbb{R}^{2}$ and let the coefficient $a_{\eta}:=\varepsilon_{\eta}^{-1}$ be as in (16). On $\varepsilon_{r} \in \mathbb{C}$ we assume that either $\operatorname{Im}\left(\varepsilon_{r}\right)>0$ or $\varepsilon_{r}<0=\operatorname{Im}\left(\varepsilon_{r}\right)$. Let $\left(u_{\eta}\right)_{\eta>0}$ be the sequence of solutions of (15) such that $u_{\eta} \stackrel{w}{\rightarrow} u$ in $L^{2}(\Omega)$ for $\eta \rightarrow 0$. We define $U \in L^{2}(\Omega)$ as the function

$$
U(x):= \begin{cases}u(x) & \text { for } x \in \Omega \backslash R, \\ N_{w}^{-1} u(x) & \text { for } x \in R,\end{cases}
$$

where $N_{w}$ is defined by (36). Then the function $\nabla U \in L^{2}(\Omega)$. The field $j_{\eta}=a_{\eta} \nabla u_{\eta}$ converges weakly to some $j$ in $L^{2}\left(\Omega ; \mathbb{C}^{2}\right)$ which is given by

$$
j= \begin{cases}\nabla U & \text { in } \Omega \backslash \bar{R}, \\ \alpha \nabla U & \text { in } R .\end{cases}
$$

Moreover, the limit functions satisfy the system

$$
\nabla \cdot\left(a_{e f f} \nabla U\right)=-k^{2} \mu_{e f f} U \text { in } \Omega,
$$

where

$$
\begin{aligned}
& a_{e f f}:=\left(\begin{array}{ll}
\alpha & 0 \\
0 & \alpha
\end{array}\right), \\
& \mu_{e f f}=N_{w} \text { for } x \in R, \\
& a_{e f f}:=\left(\begin{array}{ll}
1 & 0 \\
0 & 1
\end{array}\right), \\
& \mu_{e f f}=1
\end{aligned}
$$

$$
\text { for } x \in \Omega \backslash \bar{R} \text {. }
$$

By applying Theorem 8 for $\Omega:=B_{r_{0}}(0)$ with a large radius $r_{0}>0$, we can treat the scattering problem with an incoming wave generated at infinity. We obtain the strong convergence of the scattered field outside the metallic obstacle and we identify the limit $U(x)$ as the solution of the effective diffraction problem. We define the exterior domain outside of $R$ as $R^{\text {ext }}:=\mathbb{R}^{2} \backslash \bar{R}$.
Theorem 9 (effective scattering problem). Let the metallic gratings be given by $\Sigma_{\eta}$ (Figure 1) and the coefficient $a_{\eta}(x):=$ $\varepsilon_{\eta}^{-1}(x)$ be as in (16). Assume further that $u^{i}$ is an incident wave solving the free space equation $\nabla^{2} u^{i}=-k^{2} u^{i}$ on $\mathbb{R}^{2}$ and $u_{\eta}$ is the unique sequence of solutions to (15) such that $u_{\eta}^{s}=\left(u_{\eta}-u^{i}\right)$ satisfies (17) and that the solution sequence satisfies the uniform bound

$$
\int_{R}\left|u_{\eta}\right|^{2} \leq C
$$

Then $u_{\eta} \rightarrow U$ strongly in $L_{l o c}^{2}\left(R^{\text {ext }}\right)$ with uniform convergence for all derivatives on any compact subset of $R^{\text {ext }}$. The effective field $U: \mathbb{R}^{2} \rightarrow \mathbb{C}$ is determined as the unique solution of the upscaled equation

$$
\nabla \cdot\left(a_{e f f} \nabla U\right)=-k^{2} \mu_{e f f} U \text { in } \mathbb{R}^{2}
$$

with (17) for the scattered field $\left(U-u^{i}\right)$.

1.5.1. Interface Conditions. The homogenized equation (24) should be understood in the sense of distributions on the whole of $\mathbb{R}^{2}$. The exterior field $U \in H^{1}\left(B_{r}(0) \backslash \bar{R}\right)$ for every large radius $r$; hence its trace on $\partial R$ from outside, denoted by $U^{+}$, is a well-defined element of $H^{1 / 2}(\partial R)$. Note that as $\nabla U$ belongs to $L^{2}\left(B_{r}\right)$, the function $U(\cdot, \cdot)$ is an element of $H_{\text {loc }}^{1}(\mathbb{R})$. This helps us to define traces of $U$ on the horizontal boundary parts from the inside. Moreover, we have the information that the distributional divergence of the vector field $j=a_{\mathrm{eff}} \nabla U$ is of class $L_{\text {loc }}^{2}\left(\mathbb{R}^{2}\right)$.

We define the transmission condition on the boundary $\partial R$ of $R$ with using traces from inside and outside of $R$. We denote by superscript + (resp., by - ) traces from outside (resp., by inside); then problem (24) can be rewritten as

$$
\begin{aligned}
\nabla^{2} U+k^{2} U & =0 \quad \text { in } R^{\text {ext }}, \\
\nabla \cdot\left(a_{\mathrm{eff}} \nabla U\right)+N_{w} k^{2} U & =0 \quad \text { in } R
\end{aligned}
$$

with the transmission (interface) conditions

$$
U^{+}=U^{-} \text {on } \partial R
$$

$$
\nabla U^{+} \cdot \vec{n}=a_{\mathrm{eff}} \nabla U^{-} \cdot \vec{n} \quad \text { on } \partial R
$$

where $N_{w}$ is defined in (36).

\section{Derivation of the Effective Model}

\subsection{A Priori Estimates}

Lemma 10. For an $\varepsilon_{r} \in \mathbb{C}$ with $\operatorname{Im}\left(\varepsilon_{r}\right)>0$, let $a_{\eta}$ be defined as in (16). Then there exists a $\lambda \in \mathbb{C}$ such that

$$
\operatorname{Im}\left(\lambda a_{\eta}\right) \geq C_{0}\left|a_{\eta}\right|
$$

where $C_{0}>0$ is independent of $\eta$. 
Proof. Let $x \in \Sigma_{\eta}$ (if $\varepsilon_{r}=a+i b$ with $b>0$, then $a_{\eta}=\eta^{2}((a-$ $\left.\left.i b) /\left(a^{2}+b^{2}\right)\right)\right)$. For an arbitrary small $\delta>0$, let us define $\lambda:=-1+\delta i$ such that $\delta\left|\operatorname{Re}\left(a_{\eta}\right)\right| \leq-(1 / 2) \operatorname{Im}\left(\lambda a_{\eta}\right)$. There exists a constant $C_{0}>0$ such that

$$
\begin{aligned}
\operatorname{Im}\left(\lambda a_{\eta}\right) & =-\operatorname{Im}\left(a_{\eta}\right)+\delta \operatorname{Re}\left(a_{\eta}\right) \geq-\frac{1}{2} \operatorname{Im}\left(a_{\eta}\right) \\
& \geq C_{0}\left|a_{\eta}\right| .
\end{aligned}
$$

For $x \in \Omega \backslash \Sigma_{\eta}$, we have

$$
\operatorname{Im}(\lambda \cdot 1)=\delta \geq C_{0}, \quad \text { where } C_{0}=\delta .
$$

Lemma 11 (gradient estimate). Suppose that the solution $\left(u_{\eta}\right)_{\eta>0}$ of (15) is a bounded sequence in $L^{2}(\Omega)$; that is, $\sup _{\eta>0}\left\|u_{\eta}\right\|_{L^{2}(\Omega)}<\infty$. Then for every compactly contained subdomain $\Omega^{\prime} \subset \subset \Omega$, the following estimate holds:

$$
\int_{\Omega^{\prime}}\left|a_{\eta}\right|\left|\nabla_{x} u_{\eta}\right|^{2} d x \leq C
$$

where $C$ is independent of the scale parameter $\eta$.

Proof. Since $\bar{R} \subset \Omega$, there exists a subdomain $\Omega^{\prime} \subset \subset \Omega$ such that $\bar{R} \subset \Omega^{\prime} \subset \subset \Omega$. Without loss of generality, let us assume that $\Sigma_{\eta} \subset \Omega^{\prime}$ and take a cut-off function $\Theta \in C_{0}^{\infty}(\Omega ;[0,1])$, where $\Theta(x)=1$ on $\Omega^{\prime}$. We test (15) with $\Theta^{2}(\cdot) \bar{u}_{\eta}(\cdot)$, where $\bar{u}_{\eta}(\cdot)$ is the complex conjugate of $u_{\eta}(\cdot)$. This gives

$$
\begin{aligned}
\int_{\Omega} a_{\eta}\left|\nabla_{x} u_{\eta}\right|^{2} \Theta^{2} d x \\
=\int_{\Omega} k^{2}\left|u_{\eta}\right|^{2} \Theta^{2} d x \\
\quad-2 \int_{\Omega \backslash \Omega^{\prime}} a_{\eta} \nabla_{x} u_{\eta} \cdot \nabla_{x} \Theta \Theta \bar{u}_{\eta} d x .
\end{aligned}
$$

We employ Lemma 10 . For a $\lambda \in \mathbb{C}$, we multiply (31) by $\lambda$ and equate its imaginary part and rearrange the factors of the second integrand which will yield

$$
\begin{aligned}
& C_{0} \int_{\Omega}\left|a_{\eta}\right|\left|\nabla_{x} u_{\eta}\right|^{2} \Theta^{2} d x \leq|\lambda| \int_{\Omega} k^{2}\left|u_{\eta}\right|^{2} \Theta^{2} d x \\
& +2|\lambda| \int_{\Omega \backslash \Omega^{\prime}}\left\{\left(\sqrt{ }\left|a_{\eta}\right|\right)\left|\nabla_{x} u_{\eta}\right| \Theta\right\} \\
& \cdot\left\{\left(\sqrt{ }\left|a_{\eta}\right|\right)\left|u_{\eta} \nabla_{x} \Theta\right|\right\} d x \Longrightarrow \\
& C_{0} \int_{\Omega}\left|a_{\eta}\right|\left|\nabla_{x} u_{\eta}\right|^{2} \Theta^{2} d x \leq|\lambda| \int_{\Omega} k^{2}\left|u_{\eta}\right|^{2} \Theta^{2} d x \\
& +2|\lambda|\left[\frac{C_{0}}{4|\lambda|} \int_{\Omega}\left|a_{\eta}\right|\left|\nabla_{x} u_{\eta}\right|^{2} \Theta^{2} d x\right. \\
& \left.+\frac{|\lambda|}{C_{0}} \int_{\Omega \backslash \Omega^{\prime}}\left|a_{\eta}\right|\left|u_{\eta}\right|^{2}\left|\nabla_{x} \Theta\right|^{2} d x\right] \Longrightarrow
\end{aligned}
$$

$$
\begin{aligned}
& \frac{C_{0}}{2} \int_{\Omega}\left|a_{\eta}\right|\left|\nabla_{x} u_{\eta}\right|^{2} \Theta^{2} d x \leq|\lambda| \int_{\Omega} k^{2}\left|u_{\eta}\right|^{2} \Theta^{2} d x \\
& +\frac{2|\lambda|^{2}}{C_{0}} \int_{\Omega \backslash \Omega^{\prime}}\left|a_{\eta}\right|\left|\nabla_{x} \Theta\right|^{2}\left|u_{\eta}\right|^{2} d x,
\end{aligned}
$$

where in the second step we used Young's inequality. We see that the first integral on the r.h.s. of (32) is bounded by the $L^{2}$ boundedness assumption on $u_{\eta}$ whereas the second integral on the r.h.s. is bounded by the boundedness of $\left|a_{\eta}\right|$ and $\left\|u_{\eta}\right\|_{L^{2}(\Omega)}<\infty$. Using the fact that $\Theta(x)=1$ on $\Omega^{\prime}$, we have $\int_{\Omega^{\prime}}\left|a_{\eta}\right|\left|\nabla_{x} u_{\eta}\right|^{2} d x \leq \int_{\Omega}\left|a_{\eta}\right|\left|\nabla_{x} u_{\eta}\right|^{2} \Theta^{2} d x \leq C$, where $C$ is independent of $\eta$ and $u_{\eta}$.

2.2. An Eigenvalue Problem in the Unit Cell Y. Let us consider the eigenvalues $0<\lambda_{1} \leq \lambda_{2} \leq \cdots \leq \lambda_{n} \leq \cdots$ of the problem

$$
\begin{aligned}
& \Delta \zeta+\lambda \zeta=0 \text { on } \Sigma, \\
& \left.\zeta(y)\right|_{\partial \Sigma}=0
\end{aligned}
$$

and we denote $\left\{\zeta_{n}\right\}$ the associated normalized eigenfunctions in $H_{0}^{1}(\Sigma)$, so that $\left\{\zeta_{n}\right\}$ is an orthonormal basis of $L^{2}(\Sigma)$. Since $\varepsilon_{r} \in \mathbb{C}$ with $\operatorname{Im}\left(\varepsilon_{r}\right)>0, k^{2} \varepsilon_{r}$ satisfies the condition

$$
k^{2} \varepsilon_{r} \notin\left\{\lambda_{n}: n \in \mathbb{N}\right\} .
$$

We set

$$
\begin{aligned}
w(y) & :=\sum_{n \in \mathbb{N}} \frac{k^{2} \varepsilon_{r}}{\lambda_{n}-k^{2} \varepsilon_{r}}\left(\int_{\Sigma} \zeta_{n}(y) d y\right) \zeta_{n}(y), \\
N_{w} & :=1+\int_{\Sigma} w(y) d y \\
& =1+\sum_{n \in \mathbb{N}} \frac{k^{2} \varepsilon_{r}}{\lambda_{n}-k^{2} \varepsilon_{r}}\left(\int_{\Sigma} \zeta_{n}(y) d y\right)^{2} .
\end{aligned}
$$

Let us consider the following boundary value problem:

$$
\begin{aligned}
\Delta w+k^{2} \varepsilon_{r} w & =-k^{2} \varepsilon_{r} \quad \text { in } \Sigma, \\
w(y) & =0 \quad \text { on } \partial \Sigma .
\end{aligned}
$$

By [23, theorem 8.22], it follows that (i) $M_{w}(y)$ is a solution of (37) and this solution is unique if $k^{2} \varepsilon_{r} \neq \lambda_{n}$ for all $n$ and (ii) if condition (34) is not fulfilled then (37) has no solution. In the next theorem we will analyze the behavior of $u_{\eta}$ as $\eta \rightarrow 0$ in the sense of two-scale convergence, compare [15]. We notice that the geometry is not only periodic in the $x_{1}$ direction but it is also periodic with respect to to the cell $Y:=$ $(-1 / 2,1 / 2) \times(-1 / 2,1 / 2)$. The metal part in the cell $Y$ is given by $\Sigma \subset Y$; see Figure 2 . 
We recall that the sequnce $\left(u_{\eta}\right)_{\eta>0}$ is weakly convergent to $u \in L^{2}(\Omega)$. We define a function $u_{0}(x, y):=u_{0}\left(x_{1}, x_{2}, y_{1}, y_{2}\right)$ as

$$
\begin{aligned}
& u_{0}(x, y) \\
& = \begin{cases}u(x) & \forall x \notin R, \forall y \in Y, \\
N_{w}^{-1} u(x)(1+w(y)) & \forall x \in R, \forall y \in \Sigma, \\
N_{w}^{-1} u(x) & \forall x \in R, \forall y \in Y \backslash \Sigma,\end{cases}
\end{aligned}
$$

where $w(y)$ is a $Y$-periodic function defined in (35). We have defined $u_{0}$ in such a way that, for every $x \in \Omega$, there holds $u(x)=\int_{Y} u_{0}(x, y) d y$. We will show in next theorem that $u_{\eta} \stackrel{2}{\rightarrow} u$ as $\eta \rightarrow 0$.

Lemma 12 (two-scale limit). Let $\left(u_{\eta}\right)_{\eta>0}$, weakly converging to $u$ in $L^{2}(\Omega)$, be a sequence of solutions of (15). Then for the function $u_{0}$ defined in (38) it holds that $u_{\eta} \stackrel{2}{\rightarrow} u_{0}$.

Outside of $R$, the strong convergence $u_{\eta} \rightarrow$ u holds in $L^{2}(\Omega \backslash$ $R)$. More precisely, $u_{\eta}$ together with all its derivatives converges uniformly on every compact subset $\Omega^{\prime} \subset \subset \Omega \backslash \bar{R}$.

Proof. We divide the proof into three steps.

(i) From the assumption on $u_{\eta}$ and the estimate (30), the sequences $\left(u_{\eta}\right)_{\eta>0}$ and $\left(\eta \nabla u_{\eta}\right)_{\eta>0}$ are bounded in $L^{2}(\Omega)$. Then there exists $u_{0}: \Omega \times Y \rightarrow \mathbb{C}^{2}$ such that, up to a subsequence, $u_{\eta} \stackrel{2}{\rightarrow} u_{0}$ and $\eta \nabla u_{\eta} \stackrel{2}{\rightarrow} \nabla_{y} u_{0}$ as $\eta \rightarrow 0$. As a $Y$-periodic function, $u_{0}$ and $\nabla_{y} u_{0}$ can be extended by periodicity to all $y \in \mathbb{R}^{2}$. This shows that $\nabla_{y} u_{0} \in L_{\text {loc }}^{2}\left(\mathbb{R}^{2}\right)$ which implies that $u_{0}(x, \cdot)$ belongs to $H_{\text {loc }}^{1}\left(\mathbb{R}^{2}\right)$, in particular, in $H^{1}(\Sigma)$ and has a trace on $\partial \Sigma$. In other words, $u_{0}(x, \cdot) \in H^{1}(\Sigma) \cap C(\bar{\Sigma})$ does not jump accross $\partial \Sigma$ by trace theorem (cf. [19, theorem 5.5.1]).

Next, we investigate the coefficient $a_{\eta}=1$ on the set $\Omega \backslash \Sigma_{\eta}$. From (30), it follows that $\sup _{\eta>0}\left\|\nabla u_{\eta}\right\|_{L^{2}\left(\Omega \mid \Sigma_{\eta}\right)} \leq C<\infty$ which implies $\eta \nabla u_{\eta} 1_{\Omega \backslash \Sigma_{\eta}} \rightarrow 0$ strongly in $L^{2}(\Omega)$. Since strong convergence implies the two-scale convergence, by localisation Lemma (cf. [3]) the two-scale limit $\chi_{0}$ vanishes a.e. in $R \times(Y \backslash \Sigma)$ and in $(\Omega \backslash R) \times Y$. Due to $\nabla_{y} u_{0}=\chi_{0}$, it implies that the function $u_{0}(x, \cdot)$ is constant in $Y \backslash \Sigma$ and for $x \in R$; and it is constant everywhere for $x \notin R$. We use this $y$-independence to define a function $U \in L^{2}(\Omega)$ as

$$
\begin{aligned}
u_{0}(x, y):= & U(x) \\
& \quad \text { for }(x, y) \in(\Omega \backslash R) \times Y \cup R \times(Y \backslash \Sigma) .
\end{aligned}
$$

We note that, at this stage of the proof, $u_{0}$ and $U$ are defined as the two-scale limit of $u_{\eta}$ and by (39), respectively.

(ii) Characterisation of Two-Scale Limit for $x \in R$. We claim that, for a.e. $x \in R$, the function $u_{0}(x, \cdot)$, which belongs to $H^{1}(\Sigma)$, solves the linear boundary value problem

$$
\begin{aligned}
\Delta_{y} u_{0}+k^{2} \varepsilon_{r} u_{0} & =0 \quad \text { in } \Sigma, \\
u_{0}(x, y) & =U(x) \quad \text { on } \partial \Sigma,
\end{aligned}
$$

$$
u_{0}\left(\cdot,-\frac{1}{2}\right)=u_{0}\left(\cdot, \frac{1}{2}\right)
$$

where (40a), (40b), and (40c) hold in the distributional sense in $\Sigma$. To verify this, we choose $\varphi(x, y)=\Theta(x) \psi(y)$, where $\Theta \in C_{0}^{\infty}(R ;[0,1])$ and $\psi \in C^{\infty}(Y ;[0,1])$ a periodic function on $\Sigma$ with $\operatorname{supp}(\psi) \cap(Y \backslash \Sigma)=\phi$. Using $\varphi_{\eta}(x)=\varphi(x, x / \eta)$ as the test function in (15), we obtain

$$
\begin{aligned}
& \int_{R} \nabla \cdot\left(a_{\eta} \nabla u_{\eta}\right) \Theta(x) \psi\left(\frac{x}{\eta}\right) d x+k^{2} \int_{R} u_{\eta} \Theta(x) \\
& \cdot \psi\left(\frac{x}{\eta}\right) d x=0 \Longrightarrow \\
& -\int_{R} a_{\eta} \nabla u_{\eta}\left[\nabla \Theta(x) \psi\left(\frac{x}{\eta}\right)+\frac{1}{\eta} \nabla \psi\left(\frac{x}{\eta}\right) \Theta(x)\right] d x \\
& +k^{2} \int_{R} u_{\eta} \Theta(x) \psi\left(\frac{x}{\eta}\right) d x=0 .
\end{aligned}
$$

Passing the two-scale limit as $\eta \rightarrow 0$

$$
\begin{aligned}
& \int_{R} \nabla \cdot\left(a_{\eta} \nabla u_{\eta}\right) \Theta(x) \psi\left(\frac{x}{\eta}\right) d x \\
& +k^{2} \int_{R} u_{\eta} \Theta(x) \psi\left(\frac{x}{\eta}\right) d x=0 \Longrightarrow \\
& -\varepsilon_{r}^{-1} \int_{R} \int_{Y} \nabla_{y} u_{0}(x, y) \nabla_{y} \psi(y) \Theta(x) d x d y \\
& +k^{2} \int_{R} \int_{Y} u_{0}(x, y) \psi(y) \Theta(x) d x d y=0 \Longrightarrow \\
& \int_{R} \int_{Y}\left(\Delta_{y} u_{0}(x, y)\right) \\
& \left.+k^{2} \varepsilon_{r} u_{0}(x, y)\right) \psi(y) \Theta(x) d x d y=0 .
\end{aligned}
$$

Since $\Theta$ was chosen arbitrarily, (40a), (40b), and (40c) hold. For every $x \in R$, we write $u_{0}(x, \cdot)=U(x)(1+w(\cdot))$, where $w \epsilon$ $H_{0}^{1}(\Sigma)$ and $w(\cdot)$ is $Y$-periodic. Clearly, $w \in H_{0}^{1}(\Sigma)$ satisfies the equation

$$
\begin{gathered}
\Delta_{y} w+k^{2} \varepsilon_{r}=-k^{2} \varepsilon_{r} \quad \text { in } \Sigma, \\
w(y)=0 \quad \text { on } \partial \Sigma, \\
w(\cdot) \text { is } Y \text {-periodic. }
\end{gathered}
$$

Then, for $k^{2} \varepsilon_{r} \neq \lambda_{n}$, as shown in Section 2.2, we express $w$ uniquely in terms of the orthonormal basis $\left\{\zeta_{n}\right\}$. Note that if the condition (34) is violated, the equation in $w$ has no solution and we are led to $u_{0}(x, y)=U(x)=0$.

Therefore, to sum up, we obtain the two-scale limit as $u_{0}(x, y):=U(x)\left(1+\sum_{n \in \mathbb{N}}\left(k^{2} \varepsilon_{r} /\left(\lambda_{n}-k^{2} \varepsilon_{r}\right)\right) \zeta_{n}(y) \int_{\Sigma} \zeta_{n}(y) d y\right)$, 
provided (34) holds. Consequently, for $x \in R$, the weak limit $u$ satisfies

$$
\begin{aligned}
u(x) & =\int_{Y} u_{0}(x, y) d y=U(x) \int_{Y}(1+w(y)) d y \\
& =U(x) N_{w} \Longrightarrow \\
U(x) & =u(x) N_{w}^{-1} .
\end{aligned}
$$

Therefore the two-scale limit is given by

$$
\begin{aligned}
& u_{0}(x, y) \\
& = \begin{cases}u(x) & \forall x \notin R, \forall y \in Y, \\
N_{w}^{-1} u(x)(1+w(y)) & \forall x \in R, \forall y \in \Sigma, \\
N_{w}^{-1} u(x) & \forall x \in R, \forall y \in Y \backslash \Sigma,\end{cases}
\end{aligned}
$$

where $w(y)=\sum_{n \in \mathbb{N}}\left(k^{2} \varepsilon_{r} /\left(\lambda_{n}-k^{2} \varepsilon_{r}\right)\right) \zeta_{n}(y) \int_{\Sigma} \zeta_{n}(y) d y$.

(iii) Strong Convergence Outside of $R$. We know that $u_{0}(x, y)=u(x)=U(x)$ holds for a.e. $x \in \Omega \backslash R$ and for all $y \in Y$. Moreover, by the assumption on $u_{\eta}$ and estimate (30), we have $\left\|u_{\eta}\right\|_{L^{2}(\Omega \backslash R)}+\left\|\nabla u_{\eta}\right\|_{L^{2}(\Omega \backslash R)} \leq C<\infty$. This then implies that $u_{\eta}$, up to a subsequence, is strongly convergent to $U$ in $L^{2}(\Omega \backslash R)$ by Aubin-Lion's Lemma, compare [24]. The uniform convergence on compact subsets of $u_{\eta}$ and of all its derivatives is a consequence of the fact that $u_{\eta}$ Helmholtz equation $\Delta u_{\eta}+k^{2} u_{\eta}=0$.

With the help of Lemma 12, we can completely determine the two-scale limit of the sequence $\left(u_{\eta}\right)_{\eta>0}$ if we know the function $U(x)$ which is defined in (39). Now we collect the properties of $\vec{j}_{\eta}$, its weak limit $\vec{j}$, and its two-scale limit $\vec{j}_{0}$.

Proposition 13. Let $u_{\eta} \stackrel{w}{\rightarrow} u$ be as in Lemma 12 and $U$ be given by (39). For $\vec{j}_{\eta}=a_{\eta} \nabla u_{\eta}$, we suppose that $\vec{j}_{\eta} \stackrel{w}{\rightarrow} \vec{j}:=\left(j_{1}, j_{2}\right)$ in $L^{2}\left(\Omega: \mathbb{R}^{2}\right)$. Then $\vec{j}$ is characterized as follows:

(i) The sequence $\vec{j}_{\eta}$ converges in the sense of two scales to $\vec{j}_{0}$ which is given by

$$
\vec{j}_{0}(x, y)= \begin{cases}0 & \text { for } x \in R, y \in \Sigma, \\ \alpha^{-1} \vec{j}(x) \chi(y) & \text { for } x \in R, y \in Y \backslash \Sigma, \\ \vec{j}(x) & \text { for } x \in \Omega \backslash \bar{R}, y \in Y .\end{cases}
$$

(ii) The limit $\nabla U \in L^{2}(\Omega)$ and it holds:

$$
\vec{j}(x)= \begin{cases}0 & \text { for } x \in R, y \in \Sigma \\ \alpha \nabla U(x) & \text { for } x \in R, y \in Y \backslash \Sigma, \\ \nabla U(x) & \text { for } x \in \Omega \backslash \bar{R}, y \in Y .\end{cases}
$$

Remark 14. We would like to point out a major difference in our $j_{0}$ and the limit function $j_{0}$ in [3]. In [3], the authors worked with a rectangular metallic subpart $\Sigma$ of type $(-\gamma, \gamma) \times$ $(-1 / 2,1 / 2)$ and therefore by defining a suitable test function, they have shown that the first component of $j_{0}$ vanishes and they obtain $j_{0}=\left(0, \alpha^{-1} \partial_{x_{2}} U\right)$ for $x \in R$ and $y \in Y \backslash \Sigma$. This is clearly not the case in this paper as the metallic subpart $\Sigma$ is chosen to be sinusoidal along $y_{2}$-axis due to geometry of the metallic structure given by Figure 1. This will lead to nonvanishing componenets of $j_{0}$ and we end up having a different $j_{0}$ compared to that in [3] and hence, we will obtain a different upscaled equation.

Proof. By (30), it follows that $\vec{j}_{\eta}$ is bounded in $L_{\text {loc }}^{2}(\Omega)$ which implies that up to a subsequence $\vec{j}_{\eta}$ two-scale converges to some $\vec{j}_{0}$. The weak limit would then be given as $\vec{j}(x)=$ $\int_{Y} \vec{j}_{0}(x, y) d y$.

The Field outside of $R$. For $x \in \Omega \backslash \bar{R}, \vec{j}_{\eta}=\nabla u_{\eta}$. Then by Lemma $12, \nabla u_{\eta} \rightarrow \nabla U$ uniformly on compact subsets of $\Omega \backslash \bar{R}$. This leads to

$$
\begin{aligned}
& \vec{j}_{0}(x, \cdot)=\vec{j}(x)=\nabla U(x) \\
& \qquad \text { for a.e. }(x, y) \in(\Omega \backslash \bar{R}) \times Y .
\end{aligned}
$$

The Field in the Metal Part of $R$. We note that $\left|a_{\eta}\right| \leq C \eta^{2}$ in $\Sigma_{\eta}$ and $\left|a_{\eta}\right| \leq C$ in $R \backslash \Sigma_{\eta}$; therefore (30) gives $\left\|j_{\eta}\right\|_{L^{2}\left(\Sigma_{\eta}\right)}^{2} \leq C \eta^{2}$ and $\left\|j_{\eta}\right\|_{L^{2}\left(R \mid \Sigma_{\eta}\right)}^{2} \leq C$. This implies $\overline{\lim }_{\eta \rightarrow 0}\left\|j_{\eta}\right\|_{L^{2}\left(\Sigma_{\eta}\right)}^{2}=0$ and by [21, theorem 17], we have $\vec{j}_{0}(x, y)=0$ a.e. in $R \times \Sigma$. Moreover, $j_{0}(x, y)=j_{0}(x)=\nabla U(x)$ for a.e. $(x, y) \in R \times(Y-\Sigma)$.

Divergence of $j_{0}$. Due to boundedness assumption on $u_{\eta}$, by (18) we have $\left\|\nabla \cdot j_{\eta}\right\|_{L^{2}(\Omega)} \leq k^{2} \sup _{\eta>0}\left\|u_{\eta}\right\|_{L^{2}(\Omega)}<\infty, \forall \eta>0$. For $\Theta \in C_{0}^{\infty}(\Omega)$ and $\psi \in C_{\text {per }}^{\infty}(Y)$, we test (18) by $\Theta(x) \psi(x / \eta)$ which gives

$$
\begin{aligned}
0= & \lim _{\eta \rightarrow 0} \int_{\Omega} \eta \nabla \cdot j_{\eta} \Theta(x) \psi\left(\frac{x}{\eta}\right) d x=-\lim _{\eta \rightarrow 0} \int_{\Omega} \eta j_{\eta} \\
& \cdot\left[\nabla \Theta(x) \psi\left(\frac{x}{\eta}\right)+\frac{1}{\eta} \Theta(x) \nabla_{x / \eta} \psi\left(\frac{x}{\eta}\right)\right] d x \\
& =-\int_{\Omega} \int_{Y} j_{0}(x, y) \cdot \nabla_{y} \psi(y) \Theta(x) d x d y .
\end{aligned}
$$

Since $\Theta \in C_{0}^{\infty}(\Omega)$ is arbitrary, $\int_{Y} \nabla_{y} \psi(y) \cdot j_{0}(x, y) d y=0$ for a.e. $x \in \Omega$ which implies $\nabla_{y} \cdot j_{0}(x, \cdot)=0$ for $y \in Y$ in distributional sense. This shows that $\vec{j}_{0}(x, y)$ is independent of $y$; that is, $\vec{j}_{0}(x, y)=\vec{j}(x)$, some function in $x$ only.

Next we determine the relation between $\vec{j}_{0}$ and $U$ as shown in [3]. We define $\Xi:=\left\{\psi \in L^{2}\left(\mathbb{R}^{2} ; \mathbb{C}^{2}\right): \nabla \cdot \psi=0\right.$, $\psi$ is $Y$-periodic, and $\psi=0$ in $\Sigma\}$. We choose a test function $\varphi(x, x / \eta):=\Theta(x) \psi(x / \eta)$, where $\Theta \in C_{0}^{\infty}(R ;[0,1])$ and 
$\psi \in \Xi$. We use $\left.\vec{j}_{\eta}\right|_{\Omega \mid \Sigma_{\eta}}=\nabla u_{\eta}$ and $\nabla \cdot \varphi(x, x / \eta):=\nabla$. $(\Theta(x) \psi(x / \eta))=\psi(x / \eta) \cdot \nabla \Theta(x)$ as $\nabla_{y} \cdot \psi(y)=0$. Then

$$
\begin{aligned}
& \int_{Y} \int_{\Omega} \vec{j}_{0}(x, y) \cdot \psi(y) \Theta(x) d x d y \\
& \quad=\lim _{\eta \rightarrow 0} \int_{\Omega} \vec{j}_{\eta}(x) \cdot \psi\left(\frac{x}{\eta}\right) \Theta(x) d x \\
& \quad=\lim _{\eta \rightarrow 0} \int_{\Omega} \nabla u_{\eta}(x) \cdot \psi\left(\frac{x}{\eta}\right) \Theta(x) d x \\
& =-\lim _{\eta \rightarrow 0} \int_{\Omega} u_{\eta}(x) \psi\left(\frac{x}{\eta}\right) \cdot \nabla \Theta(x) d x \\
& \quad=-\int_{Y} \int_{\Omega} u_{0}(x, y) \psi(y) \cdot \nabla \Theta(x) d x d y .
\end{aligned}
$$

Since $\left.\psi(y)\right|_{\Sigma}=0$ and is nonvanishing in $Y \backslash \Sigma$ and by (39) it implies that $u_{0}(x, y)=U(x)$, all these lead to

$$
\begin{aligned}
& \int_{Y} \int_{\Omega} \vec{j}_{0}(x, y) \cdot \psi(y) \Theta(x) d x d y \\
& =-\int_{Y} \int_{\Omega} U(x) \psi(y) \cdot \nabla \Theta(x) d x d y \Longrightarrow \\
& \int_{Y} \int_{\Omega} \vec{j}_{0}(x, y) \cdot \psi(y) \Theta(x) d x d y \\
& \quad=-\int_{\Omega} U(x) \nabla \Theta(x) d x \cdot \int_{Y} \psi(y) d y .
\end{aligned}
$$

Therefore, $j_{0}(x, y)=\nabla U(x)$ for $(x, y) \in R \times(Y \backslash \Sigma) \cup(\Omega \backslash \bar{R}) \times Y$ and for reminder $j_{0}(x, y)=0$ for $(x, y) \in R \times \Sigma$.

Proof of (i). To conclude this part, the arguments rely on that of [3]. We consider $\vec{j}_{1}(x, y):=\left.\alpha^{-1} \vec{j}(x) \chi(y)\right|_{Y \backslash \Sigma}$. We intend to show that $\vec{j}_{1}(x, \cdot)=\vec{j}_{0}(x, \cdot)$ for almost every $x \in R$. To show this, we define a function $\vec{\psi}_{0}(y)=\vec{j}_{0}(x, y)-\vec{j}_{1}(x, y)$. We notice that (i) $\int_{Y} j_{0}(x, y) d y=\int_{Y} j(x) d y=j(x)$ and $\int_{Y} j_{1}(x, y) d y=\left.\int_{Y} \alpha^{-1} \vec{j}(x) \chi(y)\right|_{Y \backslash \Sigma} d y=j(x) \alpha^{-1}|Y \backslash \Sigma|=$ $j(x)$, (ii) $\nabla_{y} \cdot j_{0}(\cdot, y)=0$ and $\nabla_{y} \cdot j_{1}(\cdot, y)=0$, and (iii) $\left.j_{0}(x, y)\right|_{\Sigma}=\left.\left[\left.\alpha^{-1} \vec{j}(x) \chi\right|_{Y \backslash \Sigma}\right]\right|_{\Sigma}=0$. This implies that (51) holds good for $\vec{\psi}_{0}$ as well as for the conjugate $\overline{\vec{\psi}}_{0}$ of $\vec{\psi}_{0}$. Therefore using the fact that $\int_{Y} \overline{\vec{\psi}_{0}} d y=0$ from (51), we have

$$
\begin{aligned}
& \int_{Y} \int_{\Omega} \vec{j}_{0}(x, y) \cdot \overrightarrow{\vec{\psi}}_{0} \Theta(x) d x d y=0, \\
& \int_{Y} \int_{\Omega} \vec{j}_{1}(x, y) \cdot \overrightarrow{\vec{\psi}}_{0} \Theta(x) d x d y \\
& \quad=\left.\int_{Y} \int_{\Omega} \alpha^{-1} \vec{j}(x) \chi(y)\right|_{Y \backslash \Sigma} \cdot\left[\vec{j}_{0}(x, y)\right. \\
& \left.\quad-\vec{j}_{1}(x, y)\right] \Theta(x) d x d y \\
& \quad=\int_{\Omega}\left[\alpha^{-1} j(x) \cdot \int_{Y} j_{0}(x, y) d y-\alpha^{-2}|j(x)|^{2}\right.
\end{aligned}
$$

$$
\begin{aligned}
& \left.\left.\cdot \int_{Y \backslash \Sigma} \chi^{2}(y)\right|_{Y \backslash \Sigma} d y\right] \Theta(x) d x \\
& =\int_{\Omega}\left[\alpha^{-1}|j(x)|^{2}-\alpha^{-1}|j(x)|^{2}\right] \Theta(x) d x=0 .
\end{aligned}
$$

Substraction of (52a) and (52b) gives $\int_{\Omega} \int_{Y} \psi_{0}(y)$. $\overline{\vec{\psi}}_{0}(y) \Theta(x) d x d y=\int_{\Omega} \int_{Y}\left|\psi_{0}(y)\right|^{2} \Theta(x) d x d y=0$. Since $\Theta$ is arbitrary, therefore $\psi_{0}(y)=0$, which shows that $\vec{j}_{0}(x, \cdot)=\vec{j}_{1}(x, \cdot)=\left.\alpha^{-1} \vec{j}(x) \chi(y)\right|_{Y \backslash \Sigma}$. This completes the proof of part (i).

Proof of (ii). To verify the claim, let us choose $\psi(y)=\left(\widehat{e}_{1}\right.$, $\left.\widehat{e}_{2}\right)\left.\chi(y)\right|_{Y \backslash \Sigma}$ and $\Theta \in C_{0}^{\infty}(\Omega)$. Note that $\int_{Y} \psi(y) d y=\alpha\left(\widehat{e}_{1}\right.$, $\left.\widehat{e}_{2}\right)=(1-(3 / 2) \gamma)\left(\widehat{e}_{1}, \widehat{e}_{2}\right)$. Then from (51), we have

$$
\begin{aligned}
& -\alpha \int_{\Omega} U(x)\left(\widehat{e}_{1}, \widehat{e}_{2}\right) \cdot \nabla \Theta(x) d x d y \\
& =\int_{Y} \int_{\Omega} \vec{j}_{0}(x, y) \cdot \psi(y) \Theta(x) d x \\
& =\left.\int_{Y} \int_{\Omega} \vec{j}_{0}(x, y) \cdot \chi(y)\right|_{Y \backslash \Sigma} \Theta(x) d x d y \\
& =\int_{\Omega \backslash R} \alpha\left(\widehat{e}_{1}, \widehat{e}_{2}\right) \cdot \vec{j}(x) \Theta(x) d x \\
& \quad+\int_{R}\left(\widehat{e}_{1}, \widehat{e}_{2}\right) \cdot \vec{j}(x) \Theta(x) d x .
\end{aligned}
$$

It follows that $\nabla U \in L^{2}(\Omega)$ and we find also that $\vec{j}(x)=$ $\alpha \nabla U(x)$ for $x \in R$ and $\vec{j}(x)=\nabla U(x)$ for $x \in \Omega \backslash R$; that is, $\vec{j}(x)=a(x) \nabla U(x)$, where $a(x)=\alpha$ for $x \in R$ and $a(x)=1$ for $x \in \Omega \backslash R$.

\section{Proofs of Theorems 8 and 9}

Proof of Theorem 8 . The proof of Theorem 8 is a straightforward consequence of Lemmas 11 and 12 and Proposition 13. It is being shown that if, for any subdomain $\Omega^{\prime}$ with $R \subset$ $\Omega^{\prime} \subset \subset \Omega, \vec{j}_{\eta}=a_{\eta} \nabla u_{\eta}$ is bounded in $L^{2}\left(\Omega^{\prime}\right)$, then, up to a subsequence, $\vec{j}_{\eta}$ is weakly convergent to some $\vec{j}$ in $L^{2}\left(\Omega^{\prime}\right)$.

By Proposition 13, we have the relation between $U$ and $\vec{j}$; that is, the weak and the two-scale limits of $\vec{j}_{\eta}$ are given in terms of $U$; see (46) and (47). Since $\Omega^{\prime}$ is arbitrary, the results of Proposition 13 hold good in all $\Omega$. Now we obatin the limit problem by dividing the proof into two following cases.

Case 1. Let $x \in \Omega \backslash \bar{R}$; then for $\phi \in C_{0}^{\infty}(\Omega)$ from (15) we have

$$
\begin{aligned}
& -\int_{\Omega \backslash \bar{R}} a_{\eta} \nabla u_{\eta}(x) \cdot \nabla \phi(x) d x=-k^{2} \int_{\Omega \backslash \bar{R}} u_{\eta} \phi d x \stackrel{\eta \rightarrow 0}{\Longrightarrow} \\
& -\int_{\Omega \backslash \bar{R}} \int_{Y} \nabla U(x) \cdot \nabla \phi(x) d x d y \\
& \quad=-k^{2} \int_{\Omega \backslash \bar{R}} \int_{Y} U(x) \phi(x) d x d y .
\end{aligned}
$$


Case 2. Let $x \in R$; then again for $\phi \in C_{0}^{\infty}(\Omega)$ from (15) we have

$$
\begin{aligned}
& -\int_{R} a_{\eta} \nabla u_{\eta}(x) \cdot \nabla \phi(x) d x=-k^{2} \int_{R} u_{\eta} \phi d x \stackrel{\eta \rightarrow 0}{\Longrightarrow} \\
& -\int_{R} \int_{Y} j_{0}(x, y) \cdot \nabla \phi(x) d x d y \\
& =-k^{2} \int_{R} \int_{Y} u_{0}(x, y) \phi(x) d x d y \stackrel{\eta \rightarrow 0}{\Longrightarrow} \\
& -\int_{R} \int_{Y \backslash \Sigma} \alpha^{-1} j(x) \chi(y) \cdot \nabla \phi(x) d x d y \\
& \quad=-k^{2} \int_{R} \int_{Y} u_{0}(x, y) \phi(x) d x d y \stackrel{\eta \rightarrow 0}{\Longrightarrow} \\
& -\alpha \int_{R} \int_{Y} \nabla U(x) \cdot \nabla \phi(x) d x d y \\
& \quad=-k^{2} N_{w} \int_{R} U(x) \phi(x) d x .
\end{aligned}
$$

The combination of (54) and (55) gives the limit problem as

$$
\nabla \cdot\left(a_{\mathrm{eff}} \nabla U\right)=-k^{2} \mu_{\mathrm{eff}} U \quad \text { in } \Omega
$$

where

$$
\begin{aligned}
& a_{\mathrm{eff}}:=\left(\begin{array}{ll}
\alpha & 0 \\
0 & \alpha
\end{array}\right), \\
& \mu_{\mathrm{eff}}=N_{w} \quad \text { for } x \in R, \\
& a_{\mathrm{eff}}:=\left(\begin{array}{ll}
1 & 0 \\
0 & 1
\end{array}\right), \\
& \mu_{\mathrm{eff}}=1
\end{aligned}
$$

$$
\text { for } x \in \Omega \backslash \bar{R} \text {. }
$$

Here we can compare our upscaled equation with the limit problem obtained in [3], especially for $x \in R$. Due to their rectangular metallic gratings inside $R$, the component along $x_{1}$ direction vanishes; that is, the first component of $\vec{j}=\mathbf{0}$ and thus the authors obtained their upscaled equation as $\alpha \partial_{x_{2}}^{2} U=-k^{2} \mu_{\mathrm{eff}} U$.

Proof of Theorem 9. The proof is devided into three steps which are demonstrated below.

(i) Uniqueness of the Limit Problem. With a fixed incident field $u^{i}$ we will show that the limit problem (24) has a unique solution. On the contrary, let us assume that $U_{1}$ and $U_{2}$ are the two solutions of (24) and set $u=U_{1}-U_{2}$. We consider the equations satisfied by difference of two solutions as

$$
\begin{aligned}
\nabla \cdot\left(a_{\mathrm{eff}} \nabla u\right) & =-k^{2} \mu_{\mathrm{eff}} u \quad \text { in } \mathbb{R}^{2}, \\
\partial_{r} u-i k u & =o\left(r^{-1 / 2}\right) \text { for } r \longrightarrow \infty .
\end{aligned}
$$

We claim that $u(x, y)=0$ for $x \in \mathbb{R}$ and $y \in Y$. The main ingredient for this uniqueness result is Rellich's first lemma and the fact that $a_{\text {eff }}$ is real and $\mu_{\text {eff }}$ has positive imaginary part. In fact $a_{\text {eff }}$ is identity and $\mu_{\text {eff }}$ is 1 outside of $R$. Let us denote the surface of a sphere $B_{r}(0)$ of radius $r$ by $S_{r}$ (:= $\left.\partial B_{r}(0)\right)$, where $r$ is chosen so large such that $R \subset B_{r}(0)$. Let $r_{0}$ be such $r$; then by (59), we have

$$
\begin{gathered}
\lim _{r \rightarrow+\infty} \int_{S_{r_{0}}}[\underbrace{\left|\partial_{r} u\right|^{2}-k^{2}|u|^{2}}_{\text {real }}+2 k \operatorname{Im}\left(u \partial_{r} \bar{u}\right)] \\
\quad=\lim _{r \rightarrow+\infty} \int_{S_{r_{0}}}\left|\partial_{r} u-i k u\right|^{2}=0 .
\end{gathered}
$$

This gives

$$
\int_{S_{r_{0}}} \operatorname{Im}\left(u \partial_{r} \bar{u}\right) d s \leq 0 .
$$

Now we multiply (58) by $\bar{u}$ and integrate over $B_{r_{0}}(0)$. Since (58) holds only in the sense of distributions and due to possible jumps on $\Gamma_{\text {hor }}$, we approximate $\bar{u}$ by smooth functions. By divergence theorem we have

$$
\begin{aligned}
& \int_{B_{r_{0}}} \nabla \cdot\left(u a_{\mathrm{eff}} \nabla \bar{u}\right) d v=\int_{S_{r_{0}}} a_{\mathrm{eff}} u \nabla \bar{u} \cdot \vec{n} d s \Longrightarrow \\
& \int_{R} \alpha|\nabla u|^{2}+\int_{B_{r_{0}}(0) \backslash R}|\nabla u|^{2}-\int_{B_{r_{0}}(0)} k^{2} \mu_{\mathrm{eff}}|u|^{2} \\
& =\int_{S_{r_{0}}} \partial_{r} u \bar{u}=-\int_{S_{r_{0}}} u \partial_{r} \bar{u} .
\end{aligned}
$$

The surface integral on r.h.s. of (62) is well defined. This can be argued as follows: outside of $R, u$ is a solution of the Helmholtz equation $\nabla^{2} u=-k^{2} u$ and so it is analytic in the exterior of $R$. Therefore the traces of $u$ and $\partial_{r} u$ are well defined in $S_{r_{0}}$, compare [25]. Comparing the imaginary parts of (62) and investing the knowledge of $\operatorname{Im}\left(\mu_{\text {eff }}\right)>0$, then

$$
k^{2} \operatorname{Im}\left(\mu_{\text {eff }}\right) \int_{B_{r_{0}}(0)}|u|^{2}=\int_{S_{r_{0}}} u \partial_{r} \bar{u} \leq 0
$$

Therefore from (63) we have $u=0$ in $R$. Since $r_{0}$ is chosen arbitrarly, for every $r$ from (60) it follows that

$$
\int_{S_{r}}|u|^{2} d s \longrightarrow 0 \quad \text { as } r \longrightarrow+\infty
$$

Thus by Rellich's first lemma (which states that the solutions $u$ of the Helmholtz equation on an exterior domain 
satisfying property (64) vanish) we obtain $u=0$ in all of $\mathbb{R}^{2}$ which concludes the proof of the uniqueness property, compare [25].

(ii) Convergence to the Limit Problem Assuming an $L_{l o c}^{2}$ Bound. Let the radius $r_{0}>0$ be such that $\bar{R} \subset B_{r_{0}}(0)$ and set $\Omega:=B_{r_{0}}(0)$. We begin with the assumption that

$$
t_{\eta}:=\left(\int_{\Omega}\left|u_{\eta}\right|^{2}\right)^{1 / 2} \leq C \quad \forall \eta>0 .
$$

The proof basically follows as the one for Theorem 8. Using (65), up to a subsequence, passing the limit as $\eta \rightarrow 0$, we obtain that $U(x)=\left.u(x) \chi(x)\right|_{\Omega \backslash R}+\left.N_{w}^{-1} u(x) \chi(x)\right|_{R}$ solves (24).

We only need to verify the radiation condition (17). By Lemma 12 it follows that $u_{\eta}$ and $\nabla u_{\eta}$ are uniformly convergent on every compact subset of $\mathbb{R}^{2} \backslash R$. Let us choose $r<r_{0}$ such that $R \subset \subset B_{r}(0) \subset \subset \Omega$. By [25, theorem 2.4] and end remark of that theorem, we have from the Sommerfeld radiation condition that the scattered field $u_{\eta}^{s}=u_{\eta}-u^{i}$ coincides on $\mathbb{R}^{2} \backslash$ $B_{r}(0)$ with its Helmholtz representation through values and derivatives of $u_{\eta}-u^{i}$ on $\partial B_{r}(0)$. By the similar representation formula, using the values and derivatives of $U-u^{i}$ on $\partial B_{r}(0)$, we can extend $U$ into all of $\mathbb{R}^{2}$ to a solution of the Helmholtz equation $\nabla^{2} U=-k^{2} U$ outside of $R$. Thus this construction of $U$ shows that $U-u^{i}$ satisfies the Sommerfeld radiation condition. The uniform convergence of $u_{\eta} \rightarrow U$ and $\nabla u_{\eta} \rightarrow$ $\nabla U$ on $\partial B_{r}(0)$ implies the uniform convergence of $u_{\eta}$ and its derivatives on all compact subsets of exterior of $R$. Finally by uniqueness of the limit from part (i), $u_{\eta} \stackrel{w}{\rightarrow} u$ as $\eta \rightarrow 0$ for the whole sequence. This shows that the Sommerfeld radiation condition holds for $r=|x| \rightarrow \infty$. which establishes (17).

(iii) Boundedness of $t_{\eta}$. In the previous step the limit problem is obtained assuming (65) is true. We will prove that (65) holds true by the method of contradiction. We suppose that $t_{\eta} \rightarrow \infty$, up to a subsequence, as $\eta \rightarrow 0$. Now we consider the normalized sequence

$$
v_{\eta}:=\frac{1}{t_{\eta}} u_{\eta} \text { such that }\left\|v_{\eta}\right\|_{L^{2}(\Omega)}=1 \text {, }
$$

Due to linearity, $v_{\eta}$ solves the original scaterring field problem with incident field $v_{\eta}^{i}=u^{i} / t_{\eta} \rightarrow 0$ as $\eta \rightarrow \infty$. Following the proofs of Lemma 12 and parts (i) and (ii), the function $V=\left.v \chi(x)\right|_{\Omega \backslash R}+\left.N_{w}^{-1} v \chi(x)\right|_{R}$ is the unique solution of (24) and satisfies the Sommerfeld wave condition. By the construction of $v_{\eta}$, we obtain $V=0$ and therefore $v_{\eta} \rightarrow 0$ weakly in $L^{2}(\Omega)$.

For outside of $R$, the gradient estimate (30) for $v_{\eta}$ remains valid and hence, $\left.v_{\eta}\right|_{\Omega \backslash R}$ remains in a bounded subset of $H^{1}(\Omega \backslash$ $R)$. Then by Rellich compactness lemma $\int_{\Omega \backslash R}\left|v_{\eta}\right|^{2} \rightarrow 0$ as $\eta \rightarrow 0$. For inside of $R$, we use the estimate (23) on $u_{\eta}$ and since $t_{\eta} \rightarrow \infty, \int_{R}\left|v_{\eta}\right|^{2} \rightarrow 0$ as $\eta \rightarrow 0$. Therefore $\int_{\Omega}\left|v_{\eta}\right|^{2} \rightarrow 0$ as $\eta \rightarrow 0$ but this contradicts the fact that $\left\|v_{\eta}\right\|_{L^{2}(\Omega)}=1$. Thus $t_{\eta}$ has to be bounded.

\section{Transmission Properties of the Effective Layer}

By Theorems 8 and 9 we have obtained the upscaled Helmholtz equation with effective coefficients. In this section we calculate the corresponding effective reflection and transmission properties of the metallic structure.

Let the rectangle $R$ be $\mathbb{R} \times(-h, 0)$ for $h>0$. We assume planar front of waves that reaches the metallic slab $(-h<$ $\left.x_{2}<0\right)$ from above $\left(x_{2}>0\right)$. The incoming waves would be partially reflected and partially transmitted through the metallic structure. Before we proceed any further we define the following parameters:

$$
\begin{aligned}
& M=\text { amplitude of the incident wave, where } M=1 \\
& \theta:=\text { incident angle, where } \theta \in(-\pi / 2, \pi / 2) \\
& T:=\text { complex amplitude and phase shift, where } T \in \mathbb{C} \\
& A_{i}, B_{i}:=\text { complex amplitudes in the structure, where } \\
& A_{i}, B_{i} \in \mathbb{C}, i=1,2 \\
& R:=\text { complex amplitude of the reflected wave, where } \\
& R \in \mathbb{C}
\end{aligned}
$$

We write the solution $U$ of (24) as

$$
U\left(x_{1}, x_{2}\right)= \begin{cases}e^{i k\left(\sin (\theta) x_{1}-\cos (\theta) x_{2}\right)}+\mathrm{Re}^{i k\left(\sin (\theta) x_{1}+\cos (\theta) x_{2}\right)} & \text { for } x_{2}>0, \\ \frac{1}{2}\left(A_{1} \cos \left(\tau k x_{1}\right)+A_{2} \sin \left(\tau k x_{1}\right)\right)\left(B_{1} \cos \left(\tau k x_{2}\right)+B_{2} \sin \left(\tau k x_{2}\right)\right) & \text { for }-h<x_{2}<0 \\ T e^{i k\left(\sin (\theta) x_{1}-\cos (\theta)\left(x_{2}+h\right)\right)} & \text { for } x_{2}<-h,\end{cases}
$$

where $\tau:=\sqrt{ }\left(N_{w} / \alpha\right)$. We are yet to determine the coefficients $A_{i}, B_{i}, R$, and $T$ by using the interface $\left(x_{2}=0\right.$ and $\left.x_{2}=-h\right)$ and transmission conditions (see (26)).

The Transfer Matrix $M$. We will calculate a transfer matrix $M$ which basically gives a transformation relation between the solutions on the upper boundary $x_{2}=0$ and the lower boundary $x_{2}=-h$. To be precise, we define a map $M: \mathbb{C}^{2} \rightarrow$ $\mathbb{C}^{2}$ (i.e., $M \in \mathbb{C}^{2 \times 2}$ ) as

$$
M:\left(\begin{array}{c}
U(0) \\
\nabla U(0) \cdot \vec{n}
\end{array}\right) \rightarrow\left(\begin{array}{c}
U(-h) \\
\nabla U(-h) \cdot \vec{n}
\end{array}\right)
$$


where $\vec{n}$ is outer normal at $x_{2}=0$ and $x_{2}=-h$ which we choose as $(0,1)$ and $(0,-1)$, respectively. In short the matrix $M$ maps the vector $\left(U^{+}, \nabla U^{+} \cdot \vec{n}\right)=\left(U\left(x_{1}, 0+\right)\right.$, $\left.\nabla U\left(x_{1}, 0+\right) \cdot \vec{n}\right)$ on the upper boundary to the vector $\left(U\left(x_{1},-h-\right), \nabla U\left(x_{1},-h-\right) \cdot \vec{n}\right)=\left(U^{+}, \nabla U^{+} \cdot \vec{n}\right)$ on the lower boundary. As the map is seen to be linear, $M$ can be expressed as a $\mathbb{C}^{2 \times 2}$ matrix. Now we determine the transfer matrix $M$ where the two columns are obtained by $M \cdot(1,0)^{t}$ and $M \cdot(0,1)^{t}$.

Columns of $M$. To obtain the first column of $M$, we study a solution $U$ of the effective system such that $\left.U\left(x_{1}, x_{2}\right)\right|_{x_{2}=0+}=$ 1 and $\left.\nabla U \cdot \vec{n}\right|_{x_{2}=0+}=0$. The solution $U$ in the interval $(-h, 0)$ is given by $(67)$. By transmission conditions we have $\left.U\left(x_{1}, x_{2}\right)\right|_{x_{2}=0+}=\left.U\left(x_{1}, x_{2}\right)\right|_{x_{2}=0-}=1,\left.\alpha \partial_{x_{1}} U\right|_{x_{2}=0+}=$ $\left.\alpha \partial_{x_{1}} U\right|_{x_{2}=0-}=0$, and $\left.\alpha \partial_{x_{2}} U\right|_{x_{2}=0+}=\left.\alpha \partial_{x_{2}} U\right|_{x_{2}=0-}=0$. With the help of these conditions, we obtain $A_{2}=B_{2}=0$ and $(1 / 2) A_{1} B_{1} \cos \left(\tau k x_{1}\right)=1$ which gives $U\left(x_{1}, x_{2}\right)=\cos \left(\tau k x_{2}\right)$. With the help of similar transmission condition we obtain

$\left.U\left(x_{1}, x_{2}\right)\right|_{x_{2}=-h_{-}}=\left.U\left(x_{1}, x_{2}\right)\right|_{x_{2}=-h_{+}}=\cos (\tau k h)$, $\left.\partial_{x_{2}} U\right|_{x_{2}=-h-}=\left.\alpha \partial_{x_{2}} U\right|_{x_{2}=-h+}=\alpha \tau k \sin (\tau k h)$, and $\left.\partial_{x_{1}} U\right|_{x_{2}=-h_{-}}=\left.\alpha \partial_{x_{1}} U\right|_{x_{2}=-h+}=0$. This gives first column of $M$ as $(\cos (\tau k h), \alpha \tau k \sin (\tau k h))^{t}$. A similar computation by taking $M \cdot(0,1)^{t}$ in account will yield the second column of $M$ as $\left(-(\alpha \tau k)^{-1} \sin (\tau k h), \cos (\tau k h)\right)^{t}$. Thus the required transfer matrix is given by

$$
M:=\left(\begin{array}{cc}
\cos (\tau k h) & -(\alpha \tau k)^{-1} \sin (\tau k h) \\
-\alpha \tau k \sin (\tau k h) & -\cos (\tau k h)
\end{array}\right)
$$

where $\tau:=\sqrt{ }\left(N_{w} / \alpha\right)$ and $\alpha=1-(3 / 2) \gamma$.

The Transmission Coefficient. After having the matrix $M$ in hand, our next step is to calculate the transfer coefficient $T$. With the help of matrix $M$, we map the values $(U, \nabla U \cdot \vec{n})$ at $x_{2}=0+$ to the values $(U, \nabla U \cdot \vec{n})$ at $x_{2}=-h-$; that is, $(1+R, i k \cos (\theta)(-1+R)) e^{i k x_{1} \sin (\theta)}$ will get mapped to $(T, T i k \cos (\theta)) e^{i k x_{1} \sin (\theta)}$. In other words,

$$
\begin{gathered}
M \cdot\left(\begin{array}{c}
1+R \\
i k \cos (\theta)(-1+R)
\end{array}\right) e^{i k x_{1} \sin (\theta)} \\
\quad=T\left(\begin{array}{c}
1 \\
i k \cos (\theta)
\end{array}\right) e^{i k x_{1} \sin (\theta)} .
\end{gathered}
$$

Here since we are only interested in the transmission coefficient $T$, we eliminate the unknown $R$. Now we follow a simple elimination technique shown in [3] and introduce two vectors $v \in \mathbb{C}^{2}$ and $w \in \mathbb{C}^{2}$ by

$$
\begin{aligned}
v & =\left(\begin{array}{l}
v_{1} \\
v_{2}
\end{array}\right):=M \cdot\left(\begin{array}{c}
1 \\
i k \cos (\theta)
\end{array}\right) \\
& =\left(\begin{array}{c}
\cos (\tau k h)-i k \cos (\theta)(\alpha \tau k)^{-1} \sin (\tau k h) \\
-\alpha \tau k \sin (\tau k h)-i k \cos (\theta) \cos (\tau k h)
\end{array}\right),
\end{aligned}
$$

$$
\begin{aligned}
w & =\left(\begin{array}{c}
-v_{2} \\
v_{1}
\end{array}\right) \\
& =\left(\begin{array}{c}
\alpha \tau k \sin (\tau k h)+i k \cos (\theta) \cos (\tau k h) \\
\cos (\tau k h)-i k \cos (\theta)(\alpha \tau k)^{-1} \sin (\tau k h)
\end{array}\right) .
\end{aligned}
$$

Since the left hand side of (70) is $R \vec{v}$, multiplying it with $v^{\perp}$ will result in the elimination of $R$ from (70). This leads to

$$
w \cdot M\left(\begin{array}{c}
1 \\
-i k \cos (\theta)
\end{array}\right)=T w \cdot\left(\begin{array}{c}
1 \\
i k \cos (\theta)
\end{array}\right)
$$

and a straightforward computation yields

$T$

$$
\begin{aligned}
& =\frac{2 i k \cos (\theta)}{2 i k \cos (\theta) \cos (k \tau h)+\sin (\tau k h)\left(\alpha \tau k+k^{2} \cos ^{2}(\theta)(\alpha \tau k)^{-1}\right)}, \\
& =\frac{1}{\cos (\tau k h)-(i / 2) \sin (\tau k h)(\alpha \tau / \cos (\theta)+\cos (\theta) / \alpha \tau)} \\
& =\left[\cos (\tau k h)-\frac{i}{2} \sin (\tau k h)\left(\frac{\alpha \tau}{\cos (\theta)}+\frac{\cos (\theta)}{\alpha \tau}\right)\right]^{-1} .
\end{aligned}
$$

By (73), we have determined the transmission coefficient $T$ which depends on wave number $k$, height of the metallic structure $h$, the aperture volume $\alpha$, effective material parameter $\tau$, and the angle $\theta$. We note that $\tau=\sqrt{ }\left(N_{w} / \alpha\right)$, where $N_{w}$ is defined by the help of an eigenvalue problem in the metallic part $\Sigma$ and we also notice that $T$ depends on the wave number $k$ by the relation $N_{w}=N_{w}(k)$. For a rather simple $N_{w}$, the graph of $|T|^{2}$ against the wave number $k$ is shown in figure 4 in [3].

Let us focus again on the case of a material that permits perfect plasmon waves, that is, of a lossless material with negative permittivity, $\varepsilon_{r}<0$; then $N_{w} \in(0,1)$. Also $\alpha=$ $1-(3 / 2) \gamma \in(0,1)$, where $0<\gamma<(1 / 2)$. This implies that the term $((\alpha \tau / \cos (\theta))+(\cos (\theta) / \alpha \tau))$ in $(73)$ is greater than or equal to 2 . Consequently, $|T| \leq 1$ and we get $|T|=1 \Leftrightarrow$ $\cos (\tau k h)=1$. This corresponds to a resonance of the plasmon waves in the metallic structure (by solving $\nabla^{2} U=-k^{2} \tau^{2} U$ for $\left.x_{2} \in(-h, 0)\right)$ with height $h$.

We see that this effect can also be deduced from the transfer matrix $M$ of (68), since for $\cos (\tau k h)=1, \sin (\tau k h)=$ 0 and we get the transfer matrix $M=I$, the Identity matrix, corresponding to perfect transmission.

\section{Competing Interests}

The author declares that they have no competing interests.

\section{Acknowledgments}

The author appreciates the financial support provided by Chair of Analysis (at TU Dortmund) during his stay there which made this work possible. 


\section{References}

[1] S. O’Brien and J. B. Pendry, "Magnetic activity at infrared frequencies in structured metallic photonic crystals," Journal of Physics: Condensed Matter, vol. 14, no. 25, pp. 6383-6394, 2002.

[2] C. Luo, S. G. Johnson, J. D. Joannopoulos, and J. B. Pendry, "All-angle negative refraction without negative effective index," Physical Review B, vol. 65, Article ID 201104, 2002.

[3] G. Bouchitté and B. Schweizer, "Plasmonic waves allow perfect transmission through sub-wavelength metallic gratings," Networks and Heterogeneous Media, vol. 8, no. 4, pp. 857-878, 2013.

[4] J. A. Porto, F. J. García-Vidal, and J. B. Pendry, "Transmission resonances on metallic gratings with very narrow slits," Physical Review Letters, vol. 83, no. 14, pp. 2845-2848, 1999.

[5] P. Lalanne, J. P. Hugonin, S. Astilean, M. Palamaru, and K. D. Möller, "One-mode model and Airy-like formulae for onedimensional metallic gratings," Journal of Optics A: Pure and Applied Optics, vol. 2, no. 1, pp. 48-51, 2000.

[6] Q. Cao and P. Lalanne, "Negative role of surface plasmons in the transmission of metallic gratings with very narrow slits," Physical Review Letters, vol. 88, no. 5, pp. 574031-574034, 2002.

[7] T. Vallius, J. Turunen, M. Mansuripur, and S. Honkanen, "Transmission through single subwavelength apertures in thin metal films and effects of surface plasmons," Journal of the Optical Society of America A: Optics and Image Science, and Vision, vol. 21, no. 3, pp. 456-463, 2004.

[8] A. Mary, S. G. Rodrigo, L. Martín-Moreno, and F. J. GarcíaVidal, "Holey metal films: from extraordinary transmission to negative-index behavior," Physical Review B, vol. 80, no. 16, Article ID 165431, 8 pages, 2009.

[9] D. Felbacq, "Noncommuting limits in homogenization theory of electromagnetic crystals," Journal of Mathematical Physics, vol. 43, no. 1, pp. 52-55, 2002.

[10] G. Bouchitté and C. Bourel, "Multiscale nanorod metamaterials and realizable permittivity tensors," Communications in Computational Physics, vol. 11, no. 2, pp. 489-507, 2012.

[11] D. Felbacq and G. Bouchitté, "Negative refraction in periodic and random photonic crystals," New Journal of Physics, vol. 7, article 159, 2005.

[12] G. Bouchitté and D. Felbacq, "Homogenization near resonances and artificial magnetism from dielectrics," Comptes Rendus Mathematique, vol. 339, no. 5, pp. 377-382, 2004.

[13] G. Bouchitté, C. Bourel, and D. Felbacq, "Homogenization of the 3D Maxwell system near resonances and artificial magnetism," Comptes Rendus Mathematique, vol. 347, no. 9-10, pp. 571-576, 2009.

[14] G. Nguetseng, "A general convergence result for a functional related to the theory of homogenization," SIAM Journal on Mathematical Analysis, vol. 20, no. 3, pp. 608-623, 1989.

[15] G. Allaire, "Homogenization and two scale convergence," SIAM Journal on Mathematical Analysis, vol. 23, no. 6, pp. 1482-1518, 1992.

[16] G. Allaire, Two-Scale Convergence and Homogenization of Periodic Structures, School on Homogenization, Trieste, Italy, 1993.

[17] D. Cioranescu and P. Donato, An Introduction to the Homogenization, Oxford University Press, 1999.

[18] H. S. Mahato and M. Böhm, "Homogenization of a system of semilinear diffusion-reaction equations in an $H^{1, p}$ setting," Electronic Journal of Differential Equations, vol. 210, pp. 1-22, 2013.
[19] L. C. Evans, Partial Differential Equations, AMS Publications, 1998.

[20] A. Lunardi, Analytic Semigroups and Optimal Regularity in Parabolic Problems, Birkhäuser, Basel, Switzerland, 1995.

[21] D. Lukkassen, G. Nguetseng, and P. Wall, "Two scale convergence," International Journal of Pure and Applied Mathematics, vol. 2, no. 1, pp. 35-86, 2002.

[22] M. A. Peter and M. Böhm, "Different choices of scaling in homogenization of diffusion and interfacial exchange in a porous medium," Mathematical Methods in the Applied Sciences, vol. 31, no. 11, pp. 1257-1282, 2008.

[23] M. Renardy and R. C. Rogers, An Introduction to Partial Differential Equations, Springer, New York, NY, USA, 1993.

[24] J. Simon, "Compact sets in the space $L^{p}(O, T ; B)$," Annali di Matematica Pura ed Applicata, vol. 146, no. 1, pp. 65-96, 1986.

[25] D. Colton and R. Kress, Inverse Acoustic and Electromagnetic Scattering Theory, vol. 93, Springer, Berlin, Germany, 1998. 


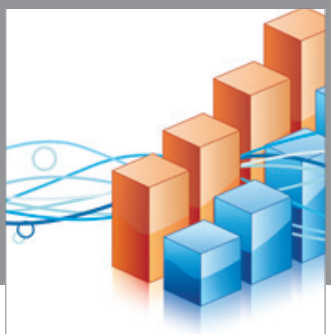

Advances in

Operations Research

vatem alat4

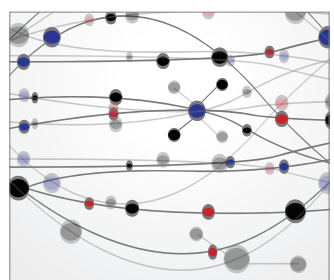

\section{The Scientific} World Journal
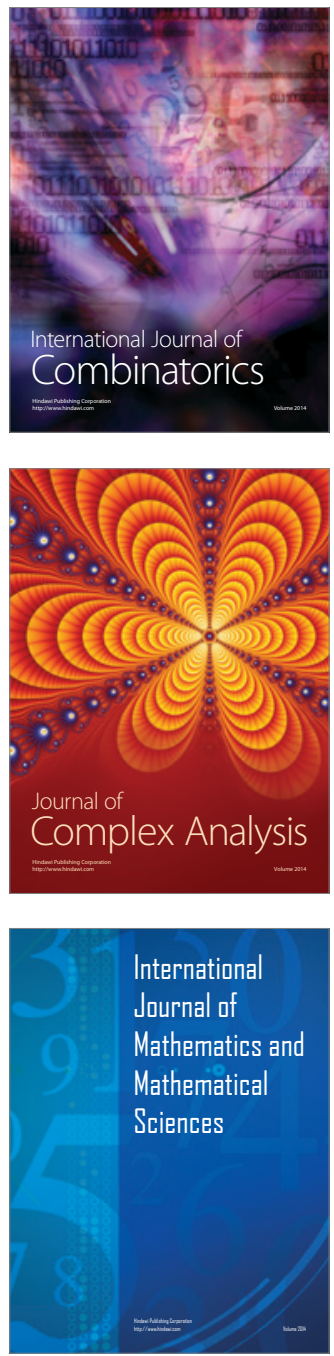
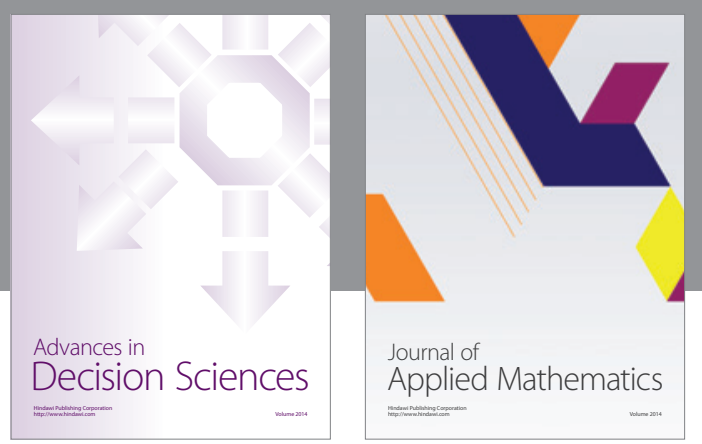

Algebra

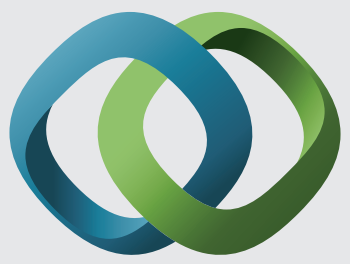

\section{Hindawi}

Submit your manuscripts at

http://www.hindawi.com
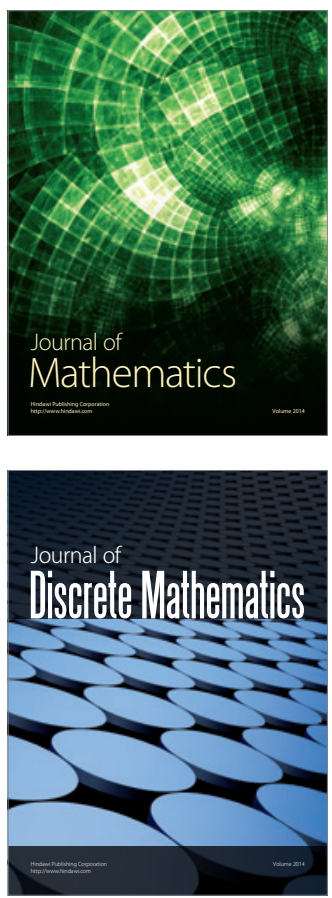

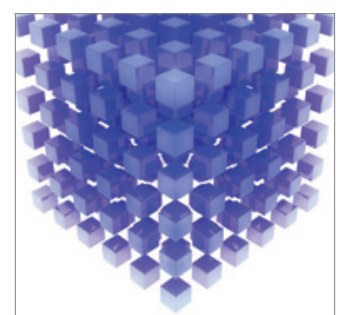

Mathematical Problems in Engineering
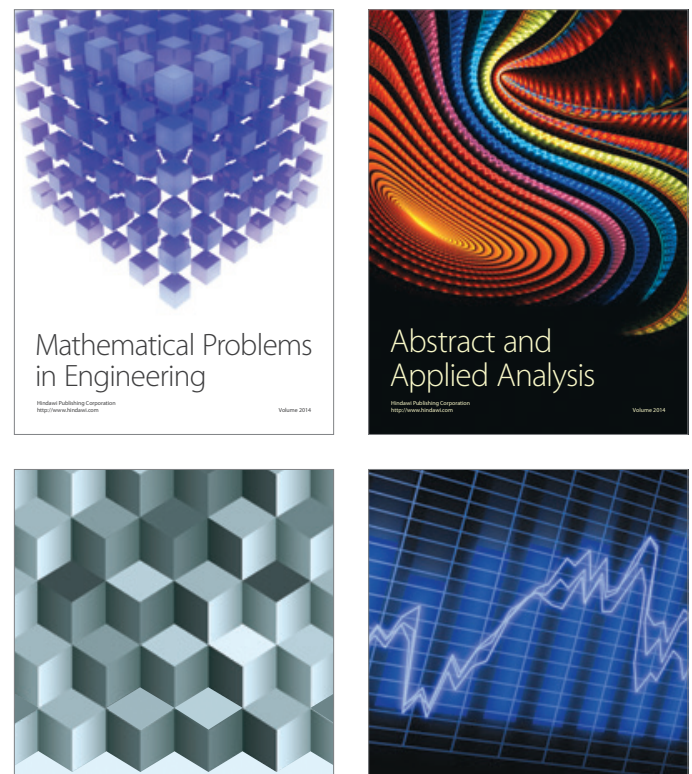

Journal of

Function Spaces

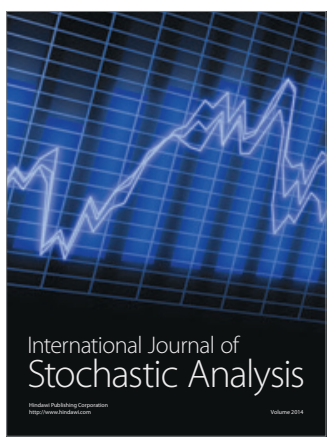

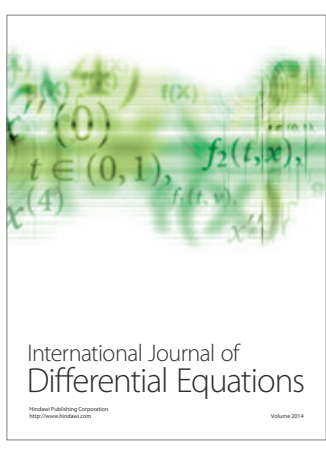
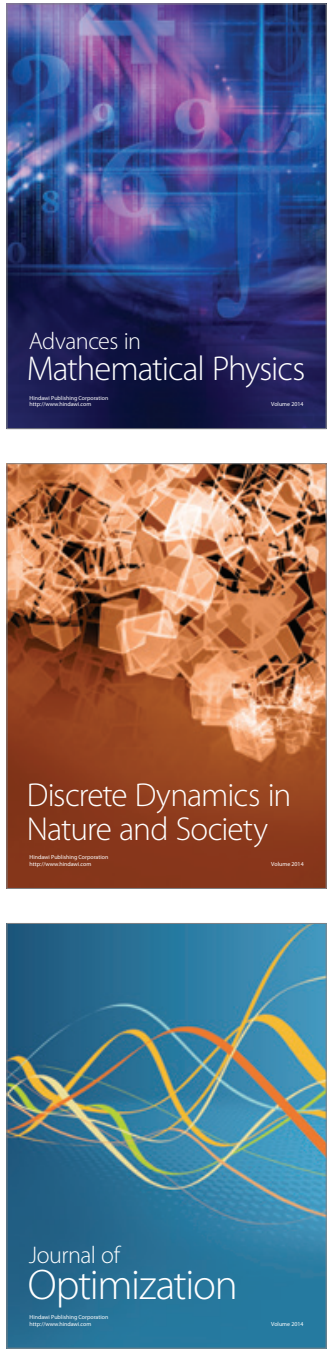